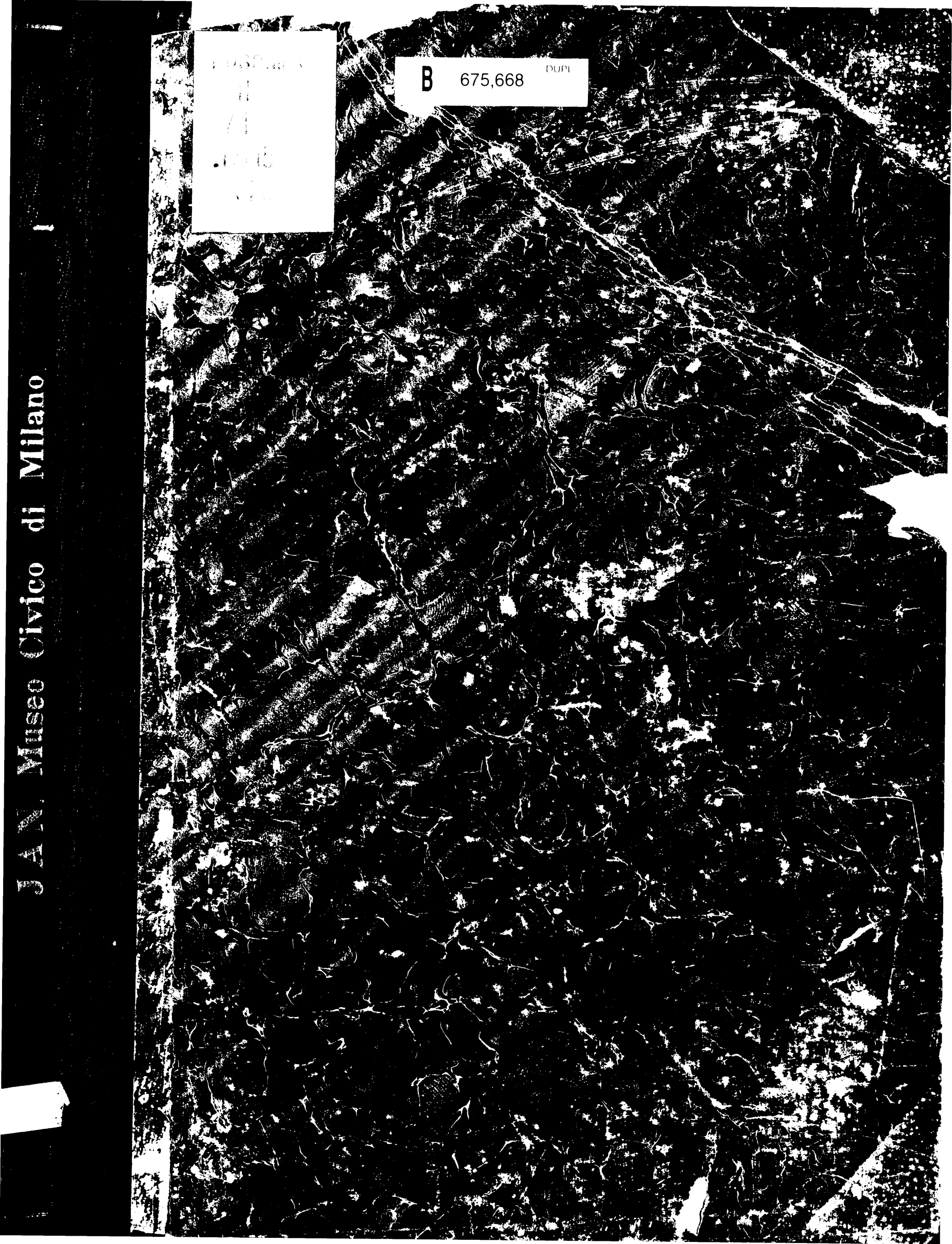



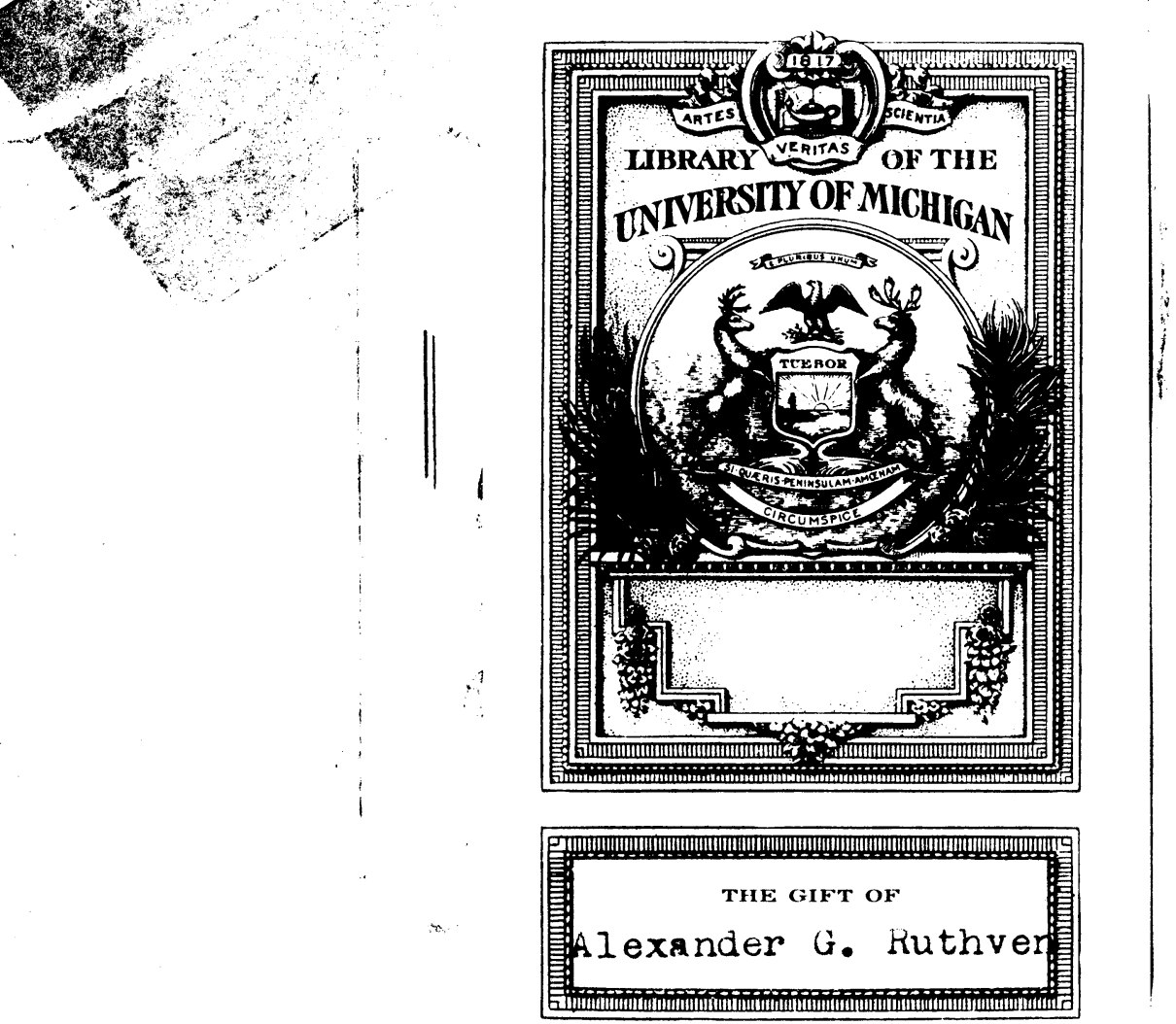


$$
\text { Whe c }
$$




\section{CENNI}

su

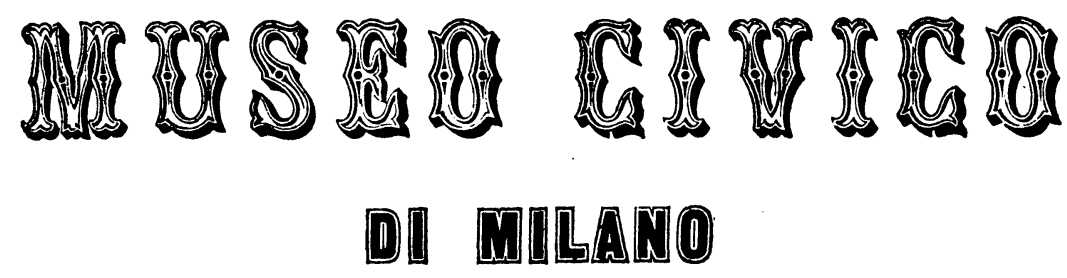

ED

\section{INDICE SISTEMATICO}

\section{DEI RETTILI ED ANFIBI}

ESPOSTI

NEL MEDESIMO

- Jan

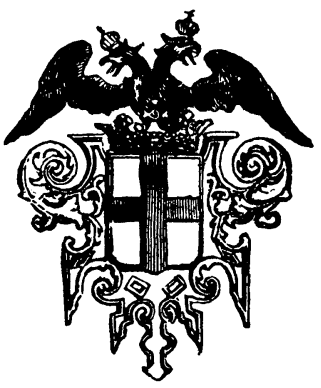

MILANO

PER LUIGI di GiACOMO PIROLA

1857 
Mureutrs

QH

71

M615

A3, 5 


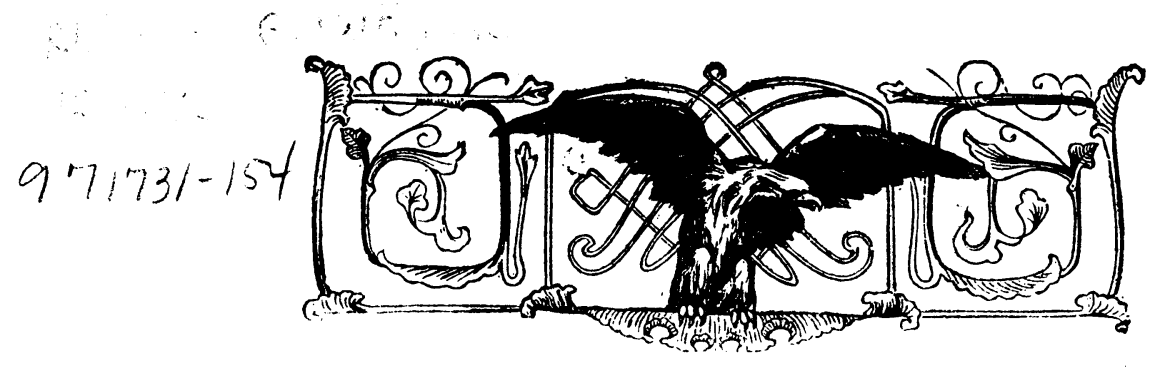

Il regolamento organico del Museo Civico adottato dal Consiglio comunale nell' anno 1845 ed approvato dal Superiore Governo nel 1845 , ha, quasi ad esordio, la breve storia di questo Istituto; cioè come sia sorto e come è passato in proprietà del Municipio di Milano.

Ora allo scopo di questo scritto nulla può venir più in acconcio che riportare qui i cenni medesimi, quali ivi si leggono e sono i seguenti:

"Il prof. Jan e Giuseppe De-Cristoforis nel 1831 conven"nero di congiungere le loro raccolte di oggetti naturali, e "che tanto quelle quanto i successivi incrementi si dovessero "ritenere di comune ed indivisa proprietà : poi il 27 Maggio "1832 stabilirono, che alla morte di uno di loro dovessero "passare in piena proprietà del superstite.

"D' allora ne fu moltissimo ampliata la suppellettile, special"mente per aggiunte fattevi con istraordinaria attività dal De"-Cristoforis nei diversi suoi viaggi.

"Venuto questi a morte sul finire del 1857 , il prof. Jan "divenne, secondo il palto, unico ed assoluto padrone di quella 
»raccolta, di ricchezza invero non ordinaria tra le private ( ${ }^{1}$ ). "Se non che il De-Cristoforis con testamento 1. ${ }^{\circ}$ Settembre "1833 aveva espresso il desiderio che, alla sua morte, il "Museo potesse divenire di proprietà civica. Conforme a tal »desiderio, il prof. Jan venne a trattative colla Congregazione "Municipale di Milano per cedere alla Città le sue ragioni sul \Museo stesso, che per tal modo passò in proprietà del Co"mune dal quale è pagato in corrispettivo al cessionario un »vitalizio di annue L. 6000 in parte a carico del Municipio se per più della metà con volontarie offerte di zelanti citta»dini $\left({ }^{2}\right)$ desiderosi di vedere arricchita la città di un tale »scientifico tesoro.

Le raccolte, oltre i libri di scienze naturali, cedute al $\mathrm{Mu}-$ nicipio nell' anno 1838 si componevano di oggetti appartenenti a tutti i tre regni della natura, ciò̀: di una copiosa suppellettile di minerali e roccie, di un ricco crbario e di una serie discreta di oggetti zoologici. In questa per altro, benchè ben provveduta fosse in varie classi degli Invertebrati, specialmente di insetti e conchiglie cosi di specie viventi come. di fossili, scarsissimo era il numero dei Vertebrati, i quali si

(1) La stima giudiziaria che ne fu fatta ne portò il valore alla somma di L. 140,000 .

(2) Di questi Signori (tutti si obbligarono per la concorrenza di annue L. 100) pongonsi quì i nomi onorevoli per ben debita lode e perchè a tutti sarà caro il conoscerli. - Ala-Ponzone marchese Filippo - Bassi nobile Carlo - Bassi nob. Elisabetta - Bassi nob. Paolo - Barbò nob. Giacomo Bellotti Felice - Borromeo conte Renato - Borromeo conte Vitaliano - Casati nob. Antonio - Casati nob. Camillo - Casati conte Gabrio - Castelbarco conte Cesare - Crivelli nob. Vitaliano - De Cristoforis nob. Luigi - De Cristoforis nob. Tomaso - De Cristoforis nob. Vitaliano - Della Somaglia conte Carlo - Giulini nob. Domenico - Giulini Della Porta conte Giorgio - Litta marchese Alessandro - Melzi nob. Gaetano - Paletta dottor Marco - Piola nob. Gabrio - Porro nob. Carlo - Taverna nob. Gaetano - Taverna conte Lorenzo - Taverna conte Paolo - Uboldo nob. Ambrogio - Uboldo noisile Giuseppe - Villa noh. Carlo - Visconti duca Uberto. 
restringevano ad alcuni mammiferi e fra questi erano principali le renne e le foche, come tuttora vedonsi esposte nel Museo. Un po' più abbondanti erano gli uccelli: pochi pesci si aveano di fiumi lombardi e del Mediterraneo, alcuni rettili solamente tra nostrali e dell' Egitto.

Ma non appena codeste raccolte vennero in proprietà e possesso del Municipio, che tosto si pose ogni pensiero ed ogni cura di supplire al difetto, sia mediante un' annua dotazione, sia accordando il Municipio sovvenzioni straordinarie, sia promovendo cambi e vendite di oggetti duplicati, di guisa che in breve tempo s' accrebbe anche la raccolta de' Vertebrati, e tanto che quando fu tenuto il Congresso scientifico in Milano nel 1844 già se ne aveva una bella serie. Ma non cessò allora di crescere, che anzi andò continuamente aumentando ognuna di queste raccolte. E cosi alla fine dell' anno 1847 videsi il numero delle spezie de' Mammiferi salire a 178, quel degli Uccelli a 1204 (e ne fu stampato il Catalogo) il numero delle specie dei Rettili era di 110; quello dei Pesci di 302.

Ed ora al chiudersi dell' anno 1856 conta il Museo $\mathbf{3 9 6}$ specie di Mammiferi, ne ha 1894 di Uccelli, 940 dei Rettili, di Pesci 890: ed in ognuna di queste quattro classi sono non pochi oggetti di gran valore per rarità e bellezza. $\left(^{1}\right)$ Ebbesi inoltre principalmente in mira di ottenere, per quanto era possibile, di ogni genere qualche specie che lo rappresentasse.

Questo notevolissimo accrescimento si ebbero nel volgere di non molti anni, le raccolte dei Vertebrati. Ma intanto per

(1) Il Principe Carlo L. Bonaparte nel suo Conspectus Systematum Mastozoologiae, Ornilhologiae, Herpetologiae el Amphibiologiae, et Jchthyologiae pubblicato nell' anno $\mathbf{1 8 5 0}$ indica il numero delle specie dei Vertebrati allora conosciute come segue :

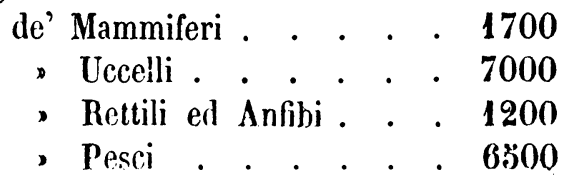


queste non furono trascurate le altre per ventura meglio gia provvedute, che pur esse vennero via via arricchite; ed a nuove si è inoltre dato incominciamento, quali sono una raccolta osteologica, e delle uova e de' nidi.

Ma se a tanto e cosi rapido arricchimento del Civico Museo non si volle meno dello spirito generoso che anima il milanese Municipio in tutto quanto ha per iscopo il lustro e il vantaggio ben inteso di questa nobile città, non è mirabile meno e men commendevole il concorso prezioso di parecchi cittadini che de' loro doni lo crebbero.

Chi vi portò il risultato di sue dotte fatiche e ricerche, chi vi depose il frutto de' suoi generosi acquisti e gli uni e gli altri gareggiando d'amore verso la bella città loro natale, di cui il Civico Museo è, non v' ha dubbio, uno de' più onorevoli ornamenti. ( $\left.{ }^{1}\right)$

(1) De' generosi cittadini che coi loro donativi contribuivano a questo rapido accrescimento del Museo, già la Gazzetta Ufficiale venne e verrà pubblicando i rispettabili nomi, ed essi pure leggonsi sui cartellini od etichette posti a dichiarazione degli oggetti donati, in attestazione della gratitudine a loro dovuta.

Intanto però si conceda di qui far menzione de' pregevoli articoli donati al Museo nel cadente anno $\mathbf{1 8 5 6} 6$, de' quali parrebber minorarsi i sensi del grato animo se si perdesse la presente occasione di esternarli. Cosi siano rese grazie al sig. Sebastiano. Mondolfi che fece bello il Museo di una superba Giraffa poco meno alta di quattro metri. Grazie al signor Ercole Turati pel raro Coccodrillo del Gange (Gavialis gangeticus) lungo anch' esso quasi quattro metri. Nè siano minori al signor Francesco Grassi che donò un bel Leone maschio ed una Tartaruga marina (Chelonia midas) di dimensione gigantesca. Ma non solo diedero i presenti : Milano ha figli le mille miglia lontani che pensarono e pensano all' onore del patrio Museo. Il milanese signor ingegnere Luigi Sada, già da più anni dimorante a San Jago del Chili che fatto aveva in altra occasione un magnifico regalo di pelli di mammiferi e di uccelli di quelle regioni, nel suo nobile intento di arricchire questo nostro istituto scientifico fece in quest', anno medesimo un nuovo invio interessantissimo che aspetto di vedere giungere in breve, essendomi per lettera annunciato già da molto tempo la partenza da Valparaiso della nave a cui venuconsegnato; esso contiene rettili, pesci, insetti ed aracnidi, ai quali 
Dopo questi brevi cenni storici del nostro Museo e dell' indole delle sue raccolte, non sarà discaro a' visitatori del medesimo e ad ogni studioso che ami giovarsi della annessa pianta per le opportune cognizioni $\mathrm{ch}^{2}$ essa può offrire, l' aver qui una succinta descrizione del locale e della collocazione

l' egregio dottor Luigi Narducci, pur milanese stabilito nel Chili, aggiunse il dono di due grandi Uccelli marini (Diomedea).

Il parmigiano dottor Enrico Morandi-Bai si è pur compiaciuto di contribuire ad arricehire le nostre raccolte, inviando dal Cairo ove ha stanza una ricca e molto interessante spedizione di rettili e piccoli mammiferi dell'Egitto e pesci del Nilo.

Il signor dottore Cristoforo Bellotti, il quale già da varj anni con zelo indefesso coltiva lo studio ittiologico, e dà luminosa prova dell' affetto che porta a questo Istituto occupandosi a vantaggio del medesimo assiduamente della classe dei pesci, ne ha pure in quest' anno fatto più dovizioso il numero delle specie con pregevoli doni.

Accenno ancor volontieri qui il dono fatto dal signor dottor Giovanni 0mboni di una bella serie di roccie della Toscana. Volontieri, dico, l'accenno, per sè stesso e perchè mi dà occasione di far noto come egli nell' amore che il trae agli studj geologici, sa pur rendere le sue occupazioni profittevoli al Museo dove dà opera all' ordinamento della Raccolta paleontologica, con manifesto ed utile ajuto.

Il Museo stesso di Parigi anch' esso concorrer volle ad arricchir il nostro molto sensibilmente, mandando il dono generoso di oltre 100 specie di rettili. Il qual egregio favore è dovuto alla squisita benevolenza del professore ed amministratore del Museo di Storia naturale esimio signor Duméril : ed essi rettili hanno per noi una tanto maggiore scientifica importanza in quanto che la raccolta del nostro museo è ordinata secondo l' Opera del donatore (Erpétologie générale par M. M. Duméril et Bibron. Paris, 1834-1855, 9 Vol.) Finalmente metto termine a questa non breve nota col registrarvi l'annunzio di una recentissima e preziosissima offerta dianzi fatta dalla signora Donna Virginia Bassi-Olivazzi, da poco vedovata del chiaro marito, il nobile Carlo Bassi, uno già de' benemeriti Conservatori di questo Istituto. Essa, indicando di compire un voto di lui, ne recava in dono al Museo tatti i libri scientifici di cui teneva gran copia, principalmente in fatto di opere entomologiche ; $e$ insieme donava la ricca raccolta de' Coleotteri europei dal medesimo posseduta. Per doppio titolo rimarrà quindi la di lui memoria in questo patrio Istituto onoranda ed onorata.

Ai quali nttli sian qui $i$ più vivi $i$ più cordiali ringraziamienti. 
degli oggetti, principalmente per norma di quelli che amino l' ispezionare le raccolte seguendo un ordine ragionato.

Sebbene, proporzionate al crescere degli oggetti, diverse siano state le provvide misure del Municipio nel procacciare tutti quelli adattamenti di cui era suscettivo il locale ove trovansi ora radunate le raccolte, sono esse tuttavia oramai cotanto stipate da far evidente la necessità o di ingrandirlo colla aggiunta di qualche nuova Galleria o di trasportare le raccolte in altro più capace ed idoneo locale, nè solo per un riguardo allo stato presente, ma vieppiù risguardando all' avvenire che fortunatamente accenna e promette un progressivo aumento delle raccolte medesime. $\mathbf{E}$ già pur delle attuali talune non poterono trovare spazio all' esposizione se non in piccolissima parte, siccome avvenne delle conchiglie fossili, ed in quelle medesime che furono esposte manca il luogo dove collocare i recenti acquisti con sistematica distribuzione.

E però caldi d' amore per un viemmaggior lustro di questo già magnifico istituto, facciamo voti che un rimedio si trovi a questo inconveniente, voti che saranno un giorno per fermo esauditi perchè gli spiriti illuminati delle autorità municipali non falliranno ai bisogni ed alla bramosa aspettazione.

Ciò premesso, cominciamo l' inspezione del Museo.

Varcata appena la soglia del medesimo presentasi a sinistra una porticina che dà adito a due stanze nelle quali (oltre a due altre divise da queste per una piccola scala) trovansi collocate le raccolte che componevano l' I. R. Gabinetto dei Minerali e Fossili posto un tempo nello Stradone di S. Teresa e dal Superiore Governo graziosamente date ad uso al $\mathrm{Mu}$ nicipio. Come il Consiglio Comunale decise nell' anno 1847 che il Civico Museo fosse trasportato definitivamente nel civico palazzo alias Dugnani, così vennero colà dapprima depositate queste raccolte nel Marzo del 1848. Se non che per la nuova temporaria destinazione data l' anno appresso al sunnominato palazzo, si dovettero di nuovo ritirare e trasportate 
furono nel locale ove trovansi di presente, occupandovi le dette quattro stanze.

Stanno nel mezzo della prima stanza quattro scaffaletti A B C D con invetriate che racchiudono una raccolta di Conchiglie fossili. Questa ha uno speciale interesse per ciò che somministrò il materiale al celebre naturalista Brocchi per la insigne sua opera "Conchigliologia subappennina ". Pel confronto delle Conchiglie fossili colle viventi egli appunto si servi delle seconde, che sono collocate nello scaffale (I) che occupa tutt' una parete della medesima stanza. Alla parete poi di rimpetto nello scaffale (II) si trova una raccolta di Roccie.

Passando da questa slanza all' attigua più grande attrae lo sguardo uno scheletro fossile di una Balena (Balaenoptera Cuvieri), lungo quasi sette metri, presso che completo. Poco discosto si osservano gli avanzi fossili di un' altra Balena, mancante in varie parti. Sopra una tavola a due scompartimenti vedonsi pezzi fossili dello scheletro d' un Elefante (Elephas primigenius), e di un Rinoceronte (Rhinoceros leptorhinus).

Si distinguono, fra quelli, porzioni delle zanne di prodigiosa grossezza e parte delle mascelle. Del Rinoceronte il pezzo più considerevole si è il cranio. Presso l' uscita verso il cortile vedonsi poi gli avanzi fossili di due Delfini.

Negli scaffali A e B conservasi una raccolta orittognostica il cui compimento trovasi negli scaffali $\mathrm{C}, \mathrm{D}$ che sono nella terza stanza.

Nello scaffale (III) sono radunati molti cristalli di quarzo nella parte sua superiore; più in basso Conchiglie fossili univalvi e bivalvi- Nel seguente (IV) si conservano Polipari fossili. Vedonsi quindi nel vicino scaffale (V) Pesci fossili ed inferiormente una serie di Radiari viventi.

Negli altri due scaffali che stanno presso l' altra parete della stanza sono Minerali e Roccie che formavano le raccolte Marzari (VI) e Carburi (VII). 
Due altri piccoli scaffali esistono nella stanzetta quarta e contengono essi pure Minerali (VIII e IX).

A chi esce dalla seconda stanza verso il cortile presentasi di fronte una porta vetrata, per la quale si entra nel vestibolo dove incominciano le raccolte proprie del Museo civico.

Uno scaffale grande a dodici imposte vetrate occupa tutta la parete (sempre due di tali imposte formano una divisione distinta con numeri). In questo scaffale si vedono Pesci e Rettili I-IV preparati a secco, e nelle divisioni V e VI alcuni Cetacei e Pinnipedi, un Delfino ed un Manato, e cinque specie di Foche, i quali tutti per la loro mole e per la ristrettezza dello spazio non si sono potuti collocare sistematicamente nelle raccolte a cui spettano.

Appesi alla vôlta del vestibolo sono lo scheletro di un Delfino, (Delphinopterus) lungo metri 3, 64, la mandibola di una Balena fossile, ed una sua scapola.

Di contro allo scaffale suddetto trovasi una stanza chiusa da vetriate in cui son collocati rettili e pesci di grandi dimensioni. Cominciando dalla sinistra, si osservano Tartarughe terrestri e marine, il Croccodillo del Nilo e del Gange, e più oltre due Pesci grossissimi; uno (Vastres) del fiume delle Amazzoni, l' altro è una Raja (Cephalopterus) presa nel Mediterraneo non lungi da Nizza.

Vicino alla scala sta una Giraffa dell' altezza di quasi \& metri.

Salita la scala trovasi di fronte la stanza ed il salone dove si hanno i libri appartenenti al Museo, distribuiti in dieci scaffali A-K secondo le materie di cui trattano. Gli scaffali A, B, C, I, K si trovano nella stanza e gli altri D, E, F, G, H, nel salone che serve di biblioteca del Museo, e dove pure ha luogo il corso delle lezioni che si suol dare ogni anno.

Quì innanzi d' imprendere la visita delle Gallerie credo opportuno notare che gli orli delle etichette che portano i nomi degli oggetti sono variamente coloriti al fine di indicare per 
lal mezzo le diverse parti del mondo cui appartiene l' individuo contrassegnato.

$\begin{array}{ccc}\text { Cosi il color nero indica l' Europa } \\ " \text { giallo } & \text { Asia } \\ " & \text { rosso } & \text { Africa } \\ & \text { verde } & \text { America } \\ & \text { celeste } & \text { Oceania }\end{array}$

Inoltre sono distinte le specie indigene nell' Italia con asterischi neri posti negli angoli delle etichette, e quelle dell' America settentrionale con quattro asterischi rossi.

Ora incomincieremo il nostro giro per le gallerie e stanze del primo piano, ma giova avvertire che questa revista superficiale la quale intraprendiamo si limita alla indicazione degli Ordini e di qualche famiglia a cui appartengono gli oggetti, e di poche specie delle più note od in qualche modo interessanti.

La prima Galleria è occupata dai Mammiferi lunghesso una parete, e dagli Uccelli lunghesso l' altra. Questi ordinati secondo l' opera di Gray (The genera of Birds), quelli secondo quella di Schreber (Die Säugethiere in Abbildungen nach der Natur) continuata da Goldfuss ed Andrea Wagner. Stanno i Mammiferi ed Uccelli disposti in una lunga fila di scaffali insiem congiunti, e distribuiti cosi che ogni divisione portante un numero comprende la larghezza sul davanti di metri 1 , 40, l' altezza di metri 2,60 e la profondità di metri 0,75. Essi sono superiormente coperti da vetrine inclinate alte metri 0,55 onde raccogliere meglio la luce che viene dall' alto per le sei lanterne praticate nella soffitta.

Cominciando dalle Scimie che insieme ai Lemurini occupano quattro scaffali, osservasi nel primo (1) di essi l' Orang-Outan ed alcune Scimie distinte per avere le braccia assai lunghe (Hilobates), nel seguente (2) il Guereza ed il Babbuino, nel terzo (3) il Mandrillo e nell' ultimo di essi (4) varie specie di Hapale, Lemuri e il singolare Tarsio spettro. Nel primo di questi scaffali si trova anche un modello in grandezza naturale 
della testa di una gigantesca Scimia, il Gorilla. A questo vicino sta pure un altro modello del busto di un Orang-Outan. Nel scaffale (ら) vedonsi il Galeopiteco, della famiglia Dermolteri, ed i Chirotter come i Cani volanti (Pteropus) e i Vampiri (Phyllostoma) ed i Pipistrelli propriamente detti, e più abbasso gli InsetTIVori di cui molte specie sono proprie dell' Europa (Ricci, Talpe, Musaragni ec.)

Negli scaffali 6-13 stanno i Carnivori. Gli Orsi, fra i quali primeggia quello delle regioni artiche, detto Orso bianco, e quello della Malaia, occupano lo scaffale (6). Nel seguente (7) vedesi in parte la famiglia delle Mustele fra le quali, molte proprie all' Europa, e fra queste pure l' Ermellino. La parte inferiore di questo scaffale è occupata da alcune specie di Cani, fra i quali l' indomabile Canis primaevus. Le Mustele stanno nella parte superiore del seguente scaffale (8) in cui sono inoltre le Volpi. Le Viverre e generi affini si osservano nello scaffale (9) in cui si trova altresi un Lupo.

Due specie di Jene sono nello scaffale (10). Viene quindi la famiglia dei Felini. Tra gli individui più notabili per bellezza sono da osservarsi la Pantera (11), la Tigre reale (12), una Leonessa (13) ed un Leone (14).

Nel medesimo scaffale e nel susseguente (1 $)$ ) trovansi Marsupiali (Didelphis, Dasyurus, Phascolomys, Petaurus, Thylacinus etc.).

La numerosa tribù dei Rosicantr è distribuita in tre scaffali 16-18. Per indicare al visitatore la collocazione di alcuni pochi tra i generi della medesima accennerò soltanto i più noti: gli Scojattoli, le Marmotte e gli Scojattoli volanti (Pteromys) nello scaffale (16). I Dipi e le Chincille nello scaffale (17) ed in questo stesso si vedono nella parte inferiore i Topi e generi vicini. Gli Istrici nella parte superiore del (18), i Castori e le Lepri nella inferiore.

Nello scaffale (19) sono gli Sdentatr, tra i quali i Bradipi, gli Armadilli ed i Formichieri. Continuano nello scaffale (20) 
gli Sdentati qui rappresentati dai Manis o Pangolini. Vi si vede quindi il singolare Ornitorinco e l'Echidna, animali della nuova Olanda.

In questo stesso scaffale si osserva un rappresentante dei Solidungoli, il Quagga. Vari Pachidermi, la più parte americani, occupano lo scaffale (21); si distinguono fra essi i Pecari (Dicotyles) il Phacochoerus Aeliani dell Abissinia ed un Tapiro.

I Ruminanti sono disposti nei successivi scaffali 22-30 e; il Daino, il Cervo (22), due Renne (23), le Antilopi (24-25), di cui una grandissima del Capo, il Gnu (27); varie Capre (28), uno Stambecco ed un Lama (29) e da ultimo un' Alpaco (50).

Prima di passare alla visita degli scaffali contenenti gli Uccelli merita un' occhiata lo scaffale isolato (A) posto a questo capo della Galleria. Quivi si osserva la Raccolta delle uova e dei nidi non ha guari incominciata.

Presso alcuni di que' nidi vedi un individuo della specie cui appartengono, affinchè maggior interesse porgano all' osservatore. Tanto delle uova quanto dei nidi il nome volgare è sottoposto al nome sistematico della specie. Passando poi in revista gli Uccelli disposti nella seconda fila di scaffali, si osservano primamente nello scaffale (31) gli Struzzı e le Ottarde e i modelli degli avanzi fossili del Todo specie ora estinta. In questo scaffale vedesi pure l' Apteryx che, come indica il suo nome, è senza ali ed ha forme assai bizzarre; questo uccello è della Nuova Zelanda.

Quattro scaffali sono occupati dai RAPACI. Gli Avoltoi fra i quali due Condor nel (32) le Aquile ed i Falchi nei due seguenti ( 33 e 34 ) ed in parte ancora del (35). Nella parte inferiore di questo vedonsi i Gufi.

I Passeri sono compresi negli scaffali 36-41. Nel primo, (36) quelli appartenenti alle famiglie Coracidi, Alcedinidi, Meropidi e Certhidi. Nel seguente (37) le Silvie, Anthus, Motacille e Tordi, di cui molti sono indigeni fra noi. Nello scaf- 
fale (38) veggonsi poi Muscicapidi ed Ampelidi e nel successivo (59) i Corvidi e Sturnidi.

Qui, interrompendo momentaneamente l' ordine sistematico nella visita, non sarà rincrescevole il contemplare gli eleganti Promeropi e Trochili collocati in due scaffaletti (I e II) sospesi presso la finestra il primo a sinistra, il secondo a destra dell' osservatore.

Continuando il giro presentasi lo scaffale (40) contenente parte della famiglia Sturnidi. I Fringillidi si trovano in parte di questo medesimo scaffale, ed in parte nel seguente (41) in cui osservansi inoltre i singolarissimi Bucerodi, caratterizzati dalle prominenze cornee a forme svariate che stanno sul loro becco.

I Rampicanti (Scansores) collocati in tre scaffali offrono pure pascolo all' esame del visitatore. I Ramphastos, detti volgarmente Mangiapepe, i Pappagalli e le Piche si osservano negli scaffali (42) e (43). Di queste ultime veggonsi pure varj individui nel seguente scaffale (44) occupato nel resto dai Cuculidi. Passando quindi all' esame dei Gallinacer, scorgesi nello scaffale (45) la ricca serie dei Colombi e delle Tortore ed i Pavoni e Meleagri. Nei due seguenti scaffali (46 e 47) si trovano Fagiani, di cui alcuni interessanti per le loro forme, inoltre Galli, Quaglie, Pernici ed altri Gallinacei ancora delle famiglie Chionidi e Tinamidi, che comprendono specie affatto esotiche.

Le Gralle occupano quattro scaffali (48-5̆1) di cui le Ardee sono collocate nei primi due (48-49) ed in parte del terzo: in questo (50) si posson vedere le Beccaccie e generi affini. I Rallidi ed alcune altre Ardee si trovano nello scaffale $(51)$.

Qui cominciano i Palmiped dei quali stanno le Anitre ed i Fenicotteri nei due primi scaffali (52 e 53). Ma conviene che qui il visitatore osservi gli Uccelli di Paradiso, la Menura lyra ed alcuni altri componenti 'la famiglia Paradiseidi che gli 
si presentano in un piccolo scaffale (B) di prospetto all' ingresso delle gallerie III e IV.

Procedendo poi nell' interrotta visita dei Palmipedi potrà vedere nello scaffale (54) gli Smerghi ed i Cigni. Nel (55) i Pinguini (Alca), i così detti Uccelli di tempesta (Procellaria), gli Albitrossi e nel successivo (56) i Gabbiani (Larus). Finalmente nei due ultimi scaffali (57) e (58) vedonsi i Generi Sula, Graculus, Atagen ed altri della famiglia dei Pellicani.

Il visitatore passi quindi alla visita della seconda e non men della prima interessante Galleria dei Rettili e Pesci.

In questa tutti gli individui sono conservati nello spirito di vino e siccome ad alcuni potrà importare di sapere in che modo si conservino in questo Museo i nominati oggetti, credo opportuno indicare le norme che si tengono per impedire per quanto si può la svaporazione dell' Alcool.

I vasi di vetro che contengono gli oggetti sono cilindrici od ovali con orlo smerigliato e si chiudono con un disco di vetro parimente smerigliato. Il coperchio o disco si fa combaciare colla bocca del vaso mediante un leggero strato di un miscuglio di cera veneta parti quattro, grasso di cervo parti quattro, spermaceta parti due, e grasso di majale parti due. Per meglio assicurare la perfetta chiusura e per una certa eleganza si suol poi cingerne l' orlo con una sottile striscia di stagnola.

Ma prima di passare all' esame dei Rettili sistematicamente disposti piaccia al visitatore di osservare nello scaffale alla di lui sinistra segnato (A) vari grossi Pesci e Sauri che per la loro dimensione non, si sono potuti collocare al loro posto rispettivo.

I Cheloni o Tartarughe occupano lo scaffale (I), ma solo in parte si sono quì raccolte; il visitatore avrà già vedute nel vestibolo individui colossali de' medesimi ed altri ancora ne vedrà nella attigua stanza seconda.

Nello scaffale (II) cominciano i Sauri, compresi i Coccodrilli 
(appena rappresentati da giovani individui ed a cui riguardo si ripete l' osservazione, fatta per le tartarughe), i Camaleonti, gli Ascalaboti, i Varani (genere di cui quasi tutte le specie note possiede il nostro Museo, ma i più sono preparate a secco e stanno nella sovraindicata seconda stanza), e parte della famiglia delle Iguane.

Nel (III) sono molt' altre specie della famiglia delle Iguane tra le quali si annoverano i Basilischi e varj Draghi volanti, la cui sola ispezione basta a far rigettare le favole di cui furon l' oggetto. Per la forma singolare sono da osservarsi le Phrynosome ed il Moloch horridus della Nuova Olanda con cui terminano gli Iguanidi. Nello scaffale IV le numerose Lucertole vi fan mostra di belle serie di varietà, specialmente delle nostrali.

Nello scaffale seguente (V) vedesi la famiglia dei Chalcidi e dei Scincoidi, nella quale alcuni generi o per lo stato rudimentale o per la mancanza di zampe fanno un passaggio al terzo ordine dei Rettili, quello dei Serpenti, la cui bella e ricca raccolta occupa il resto degli scaffali destinati ai rettili (VI-XII). Alcuni per altro di considerevole dimensione non si trovano nel posto che loro dovrebbe spettare e sono dei Generi Python, Boa, Xenodon, Naja ed altri. Essi stanno nei due scaffali ad una sola imposta segnati (B e C). I due ultimi scaffali nella fila opposta (XI e XII) contengono più specialmente i Serpenti velenosi.

Gli Anfibi sono collocati nei due susseguenti scaffali (XIII e XIV). Gli altri sette (I-VII) distinti con cartellini verdi sono occupati dai Pesci.

Retrocedendo verso il fondo della Galleria si passa in una stanza nella quale è posta una Raccolta osteologica. Il visitatore vi potrà osservare nello scaffale (I) gli Scheletri di varie Scimie, crani di Quadrumani e Carnivori e più abbasso crani di Ruminanti e Pachidermi e tra questi quello d' un Ippopotamo. Nello scaffale (II) gli scheletri completi di varj Carnivori, 
nove modelli di crani delle differenti Razze umane e crani di molti Rosicanti. La parte inferiore di questo scaffale è occupata dal cranio di un Bue e dagli scheletri di varj Carnivori tra i quali primeggia una Jena.

Nel successivo scaffale (III) sull' altra parete si vedono gli scheletri di varj Sdentati come pure quello d' un Leone e d' una Foca (Phaca barbata).

Nel (IV) sono gli scheletri di varj Rosicanti quello d' un Delfino comune e di un Coccodrillo (Alligator lucius).

Nel (V) scheletri di Uccelli rapaci e Passeri: vi è anche uno Struzzo ed un Fenicottero e nel (VI) scheletri di molte sorta di Pesci di cui varj sono esposti anche sulla prima delle tavole (A) poste nel mezzo della stanza; sulla medesima sono inoltre gli scheletri di varj Pipistrelli, d' un Sorice e d' uno Scojattolo, e dieci preparati anatomici per dimostrare la varia forma degli ossicini dell' orecchio nei Mammiferi, e due modelli in cera rappresentanti il cuore e le branchie d' un Pesce.

Nella seconda tavola (B) vedonsi gli scheletri di più sorta di Serpenti, dei quali sono da osservarsi una Naja e due Crotali; inoltre due Tartarughe e varj Sauri (Salvator, Uromastix, Chamaeleo), lo scheletro del Proteo anguino, inoltre quelli di varie Rane, Rospi e Salamandre. Sono appesi al muro dalla parte delle finestre ed in faccia agli scaffali III e IV due quadri. Nel primo alla diritta si vede l' anatomia osteologica di una Testuggine, ogni pezzo dello scheletro è distinto con numeri; nel secondo il cranio d'un Pesce (Gadus morhua) anch' esso separato nei suoi varj pezzi.

Presso lo scaffale VI vi sono tre altri quadri. I due superiori contengono le mascelle fornite dei denti di grandi specie di Pesci plagiostomi. L' inferiore contiene la classificazione del gruppo dei Ciprini secondo la forma dei denti proposta da Heckel.

Da questa stanza si progredisce nella seconda in cui sono 
esposti Rettili e Pesci preparati a secco e le ossa fossili di Mammiferi e Uccelli in cinque scaffali. Cominciando ora l' ispezione, il primo scaffale (A) che presentasi a diritta entrando contiene esclusivamente ossa di Mammiferi di specie ora estinte. Vi sono esposti quei del Mastodonte, del Megaterio, di Rinoceronti ed altri; più abbasso il Dinoterio e varj denti di Pachidermi; notisi però che in parte si è dovuto supplire coi modelli in gesso. Nello scaffale in fronte a questo (B), vedonsi pure gli avanzi fossili di Carnivori, Cetacei, Ruminanti, Rosicanti ec. Inferiormente si osserva la testa dell' Orso delle caverne (Ursus spelaeus), superiormente i modelli di due ova gigantesche e parti della gamba dell Epiornis. In mezzo alla stanza sono due tavole. La prima (I) presso lo scaffale B contiene una bella serie di Testuggini poste tutt' intorno alla tavola; in mezzo sono varj Serpenti, tra i quali un Pitone e due Crotali, uno del sud, l' altro del nord dell' America, vi si osservano altresi varj Batraci, il Siren lacertina e le spoglie di varj Serpenti.

Sulla seconda tavola (II) si offrono allo sguardo molti grossi Sauri tra i quali primeggiano i Varani e le Iguane.

Sono appesi al muro due quadri contenenti i modelli in cera dello sviluppo della Rana rossa.

Volgendo le spalle agli or ora citati quadri veggonsi sulla parete in faccia tre scaffali C, D, E; quello di mezzo (D) contiene tre Coccodrilli, varie Testuggini, ed in una custodia a vetri $\mathrm{i}$ modelli in cera dell' apparato velenigeno, molto ingrandito dal naturale, della Vipera nostrale. Appresso vedonsi due grossi Serpenti preparati a secco (Epicrates, e Morelia), nel medesimo scaffale sono anche tre vasi con Serpenti nello spirito di vino, che non si son potuti collocare nell' attigua Galleria. D' ambo i lati di questo scaffale sono due altri (C E) contenenti Pesci, tutti preparati a secco.

Da questa stanza si passa nella vicina terza, nella quale sono tre scaffali $(1,2,5)$ tutti occupati da Pesci petrefatti, alcuni 
quà e là appesi al muro, come pure si vedono gli avanzi ed i modelli di Ittiosauri e Teleosauri e la testa di un Batraco, il Labyrinthodon.

Uscendo da questa stanza, si ritorna nella Galleria dei Mammiferi dalla quale, passando per la stanza ove conservasi l' Erbario, si entra nella terza Galleria.

Qui si presenta una serie di tavole che occupano longitudinalmente la Galleria; queste tavole sono divise in quaranta compartimenti e contengono i principali rappresentanti dei generi degli Insetti; essi vi si trovano cosi distribuiti: 1-3 Ditteri, 4-9 Lepidotteri, 10-11 Emitteri, 12 Neurotteri, 13-14 Imenotteri, 15-16 Ortotteri, 17-40 Coleotteri.

A chi fa la rassegna degli scaffali situati in questa stessa galleria può giovare l' indicazione che il primo segnato A contiene racchiusa parte della raccolta dei Coleotteri e ne' vasi su di esso posti sono Acalefi, Entozoi e Radiari nello spirito di vino; presso a questo e nell' angolo della Galleria c' è uno scaffaletto (1) appeso contenente pure Coleotteri, su questo si trovano due campane di vetro con Radiari; dietro a queste $\grave{e}$ in alto sul muro un quadro con varie Asterie.

Vicino a questo scaffaletto (1) un altro scaffale segnato (I) contenente la continuazione della raccolta dei Coleotteri porta nella parte sua superiore una vetrina in cui sono esposte Farfalle esotiche.

Lo scaffaletto seguente (2) contiene Imenolteri e sopra di esso sono due cassette con vetri, in uno delle quali si vede il nido della Vespa cartaria, nell'altra il tessuto ed i bozzoli d' una specie di Bombice.

I due scaffali che vengono in appresso (II e III) contengono il primo la sequela dei Coleotteri, il secondo Lepidotteri europei, ambidue portano in esposizione Farfalle esotiche. Lo scaffaletto (3) contiene Ditteri e superiormente porta una cassetta con un voluminoso Vespajo.

Lo scaffale (IV) contiene Emitteri e superiormente al medesimo 
vedonsi Farfalle esotiche notturne. Lo scaffaletto (4) contiene Neurotteri e superiormente sta una cassetta col modello in cera assai ingrandito di un Acaro (Ixodes ricinus).

Fra le raccolte de' differenti Ordini d' Insetti che possiede il Museo e di cui si è ora fatto menzione, la più importante è quella dei Coleotteri di cui si hanno più di 9000 specie. I susseguenti scaffali (V, VI, VII, VIII) contengono Cirripedi, Trilobiti, Echinodermi, Polipari e Rizopodi (nello scaffale (V) è pur rinchiuso un piccolo numero di Insetti fossili), rappresentanti dei generi dei medesimi si trovano esposti nella parte superiore vetrata.

Di prospetto allo scaffale A al termine della galleria se ne vede un altro (B) che contiene Conchiglie fossili; sul medesimo sono esposti Molluschi, Ragni e Miriapodi conservati nello spirito di vino.

Prima di partire da questa galleria si possono osservare anche varj quadri appesi alle pareti della medesima, di cui XIV, portanti il loro numero progressivo, contengono Coleolteri europei, in due, di cui uno presso l' ingresso della galleria, l' altro presso la sua uscita sono larve di varj Lepidotteri, in quattro altri sotto ai quadri XI-XIV Farfalle esotiche; finalmente verso la metà della galleria si scorgono appesi presso la stufa due quadri contenenti modelli e preparati dello sviluppo e della anatomia del Baco da seta.

Entrato nella attigua stanza veggonsi Crostacei esposti sulla tavola in mezzo (che ha dieci compartimenti) e sopra gli scaffali che fanno continuazione allo scaffale $B$ della terza Galleria (C, D, E, F), sullo scaffale (G) sono pure Crostacei e Cefalopodi nello spirito di vino.

Negli scaffali B (che sta nella terza Galleria) C, D, E, F (che sono in questa stanza), è custodita la Raccolta paleontologica dei Molluschi; essa è ricca di circa mille specie appartenenti di preferenza ai terreni terziari e specialmente ai terreni pliocenici dell' Appennino ed ai bacini lerziari di Parigi, 
di Bordeaux e di Vienna. Nello scaffale $\mathbf{G}$ sono rinserrate Conchiglie terrestri e fluviali.

Tra le due finestre in un gran quadro si vede un Crostaceo del mare del Giappone (Macrocheira) e dallo stesso lato quattro minori quadretti nei quali sono pure Crostacei di cui lo scheletro esterno è separato e nelle sue parti. Presso l' uscio a muro che mette ad uno stanzino riservato sono un quadretto di piccoli Crostacei ed un altro di Crostacei fossili.

Progredendo nella visita si passa nella attigua stanza in cui sono Invertebrati fossili. La tavola nel mezzo della medesima, divisa in dieci compartimenti, offre una serie di Conchiglie fossili dell' ordine dei Brachiopodi disposte zoologicamente e portanti etichette variamente colorite a distinzione dei terreni nei quali si rinvengono. Solto di essa veggonsi varie

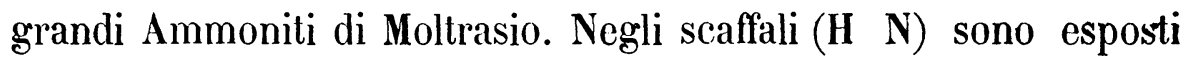
Cefalopodi fossili; qui si osserva in ispecie una bella serie di Ammoniti, nel seguente (L) vedonsi dei Polipari di specie tuttora viventi: ai due lati di questo scaffale osservansi, difese da rete di ferro; Ammoniti ed Orthoceratiti.

Sopra i due altri scaffali (I M) sono esposti avanzi fossili di Conchiglie essi poi contengono rinchiuse Conchiglie marine: (lanto queste quanto le terrestri e fluviali che sono nello scaffale $\mathrm{G}$ dell' antecedente stanza si dovrebbero in gran parte inserire nelle raccolte esposte nella Galleria IV). Tra le due finestre osservasi il modello in gesso di un Pentacrinites subangularis.

Delle altre tre piccole stanze l' ultima serve di Magazzino, e nelle prime, due riserbate ad uso dell' Ufficio, hannosi molti quadretti contenenti insetti che già facevano parte della raccolta del sacerdote D. Bernardo Marietti, (alla quale pure appartenevano i XIV quadri dei Coleotteri nella terza Galleria) ceduta dai suoi fratelli al Municipio dopo la di lui morte, e vi è pure la raccolta malacozoologica del defunto nobile Carlo Porro, della quale la onorevole famiglia fece dono prezioso 


\section{2}

e generoso al Museo: di quel Porro, io dico, che mai non mi ricorre al pensiero se non accompagnato da mestissimo desiderio, dalla rimembranza delle sue doti amabili e peregrine. Vi sta inoltre la raccolta dei Coleotteri testè regalata dalla nobile .Signora Virginia Bassi-Olivazzi, ed accennata più sopra nella nota (1) pag. 6.

Retrocedendo, dalla terza Galleria si passa di nuovo per la stanza dell' Erbario, ed ecco si presenta alla sinistra la quarta Galleria che offre la raccolta delle Conchiglie marine fluviali e terrestri non che le Raccolte mineralogiche e geognostiche.

La raccolta delle Conchiglie è disposta sopra una serie di tavole divise in ottantotto compartimenti. I primi undici comprendono i generi delle Conchiglie viventi e fossili. Le specie poi si trovano cosi distribuite: 12-27 le terrestri, 28-39 le fluviali, 40-88 le marine.

Chi desiderasse occuparsi di qualche genere in particolare, troverà appeso fra la prima e la seconda finestra un indice sistematico ed alfabetico dei generi delle conchiglie, ed una tabella delle specie dei minerali, colla indicazione della loro rispettiva collocazione.

I due scaffali C e D contengono Conchiglie di grandi dimensioni: nel primo stanno univalvi e nel secondo bivalvi.

Se per avventura paja singolare la nuova materiale disposizione delle tavole (che stanno tanto in questa quanto nella terza Galleria ed in tutte le altre stanze, tranne quella ove conservasi l' Erbario) portanti gli oggetti diversi in esposizione, io spero che almeno non sembri a nessuno irragionevole $e$ disappensata. Ognuno infatti potrà notare facilmente che con que' piani orizzontali e rivolti verso il muro ai quali se ne congiungono altri inclinati verso le finestre ho forse raggiunto il moltiplice intendimento che mi era proposto, di utilizzare lo spazio, di non perder luce, di evitare le troppo frequenti interruzioni nella serie degli oggetti esposti, e di lasciar il più possibilmente libero il campo ai visitatori di aggirarsi per le gallerie e stanze senza troppa confusione. 
La Raccolta dei Minerali è disposta secondo il metodo di Beudant in qualche parte modificato. Essa è custodita in ventidue scaffali, sopra tre ordini di assi leggiermente inclinate. A queste sovrastano due altre assi attaccate al fondo dello scaffale per i pezzi voluminosi appartenenti alle specie già deposte nella serie continua delle tre assi sopra indicate, e ciò perchè non disturbino col loro soverchio volume la distribuzione della serie principale composta di pezzi d' uniforme e mediocre grandezza. La raccolta è composta di 2770 pezzi distribuiti in circa 370 specie. Primeggiano in essa gli esemplari magnifici di zolfo, celestina ed arragonite modificata di Sicilia che si trovano nello scaffale $\mathrm{A}$, in cui esiste un rilievo del Vesuvio. Lo scaffale 22 contiene le aggiunte che andrebbero intercalate al loro posto rispettivo.

Sonovi poi nello scaffale B varj pezzi di Malachite, Stibina, Rame, Calcedonia, Gesso ed altri, non che due saggi per dimostrare le teorie di Gorini sulla origine delle montagne.

Le Raccolte geologiche esposte e che trovansi sotto la raccolta dei minerali nei 22 scaffali sono le seguenti: Quella del Piemonte e della Sardegna (1), dei dintorni di Parigi (2), del Vicentino e dei Colli Euganei (5-6), del Vesuvio e Campi Flegrei (7-8), della Provincia di Bergamo (10), della Provincia di Como (14-16), dell' Isola d' Elba (13), della Germania (10-15); una raccolta di roccie in serie orittognostica si trova negli scaffali 17-18, e vi sono inoltre una di Roccie dell' Egitto (19), una del Tirolo $(20,21)$.

Nelle cassette delle tavole in mezzo di questa Galleria, sono racchiusi oltre i Minerali duplicati, una Raccolta geognostica e paleontologica dell' Istria, una di Val di Fiamme ed una deî Monti Pisani e del Campigliese.

Nell' ultima stanza conservasi l' Erbario di più di 17,000 specie di Piante disposte secondo il sistema di Linneo contenuto negli scaffali A-F, lo scaffale $G$ contiene poi le Piante fossili. Tra queste si osservano le Foglie fossili dell' Oltrepò. 


\section{4}

le Fucoidi Briantee (Cretacee) e i Vegetabili del terreno carbonifero di Rive, di Giers e della valle di S. Étienne.

In uno scaffaletto appeso sono custoditi varj legni di piante indigene e forestieri, e presso l' uscita uno scaffale apposito contiene una raccolta di Semi disposti secondo le famiglie delle piante.

La ristrettezza del locale (il quale sebbene per sè stesso disteso, è sfortunatamente angusto alla copia ognor crescente degli oggetti) non permise sempre di presentare essi oggetti in quel modo che sarebbesi desiderato al fine di non interrompere la loro collocazione sistematica. Cosi, a cagion d' esempio, fu forza ricoverare in una medesima Galleria le Conchiglie ed i Minerali, convien che passi tre volte per la stanza dell' Erbario chi voglia ispezionare le raccolte del Museo per intero; e di parecchie raccolte non fu possibile far mostra, per mancanza di spazio per cui giacciono racchiuse negli scaffali, siccome è stato indicato a suo luogo. Ma questi sono inconvenienti inevitabili quasi in ogni edifizio che serve per modo provvisorio, e che sarebbero tolti in altro il quale venisse appositamente eretto 0 stabilmente adattato per servire allo scopo.

Con questa rapida e succinta indicazione delle cose serbate nel Museo non altro ebbesi in mira fuorchè di facilitare l' intendimento e l'uso della qui annessa pianta nella quale si trovano indicati tutti gli scaffali con lettere 0 con numeri.

I Cataloghi poi, che verranno man mano pubblicati in avvenire offriranno ai visitatori l' agio di meglio ispezionare quelle raccolte di che essi abbiano fatto uno studio particolare o di cui bramino avere un elenco sistematico. E spero che non siano quindi per mancare le appendici ai Cataloghi stessi, al fine di tener gli studiosi in corrente nella conoscenza delle nuove aggiunte, che il tempo e le cure apporteranno in aumento del Museo medesimo. 
Ora avendo con questi brevi cenni fatto noto l' origine, il progresso e la presente condizione del civico Museo, non so tenermi dall' esprimere qui il mio non ignobile voto che piaccia a' cittadini di onorare di loro visite frequenti questo patrio Istituto, e, secondo è nel poter di ciascuno, arricchirlo di loro favori seguendo le generose traccie di molti i quali già ne dieder l'esempio. Potrà essere stimolo per molti a nobilissimi studi, e ad ogni modo, essendo esso perenne monumento di lustro alla città, rimarrà sorgente a' cittadini di vive e non volgari compiacenze.

Milano 31 Dicembre 1856.

G. JAN.

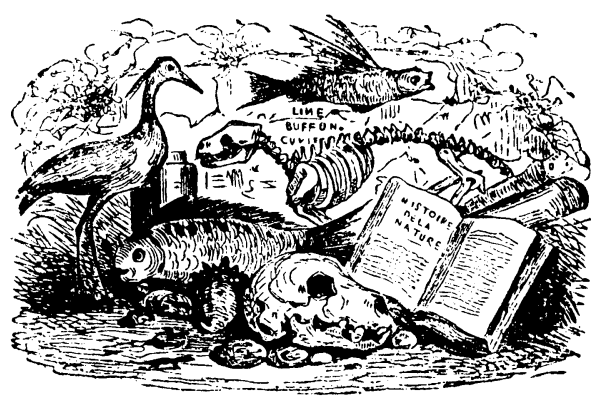




\title{
INDICE SISTEMATICO
}

\author{
DEI \\ RETTILI ED ANFIBI \\ ESPOSTI
}

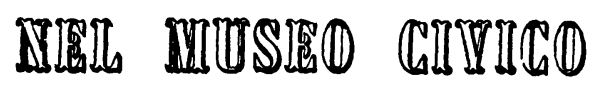

DI MILANO 



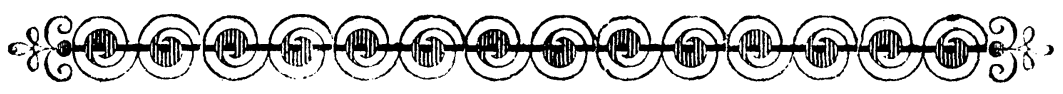

Quando le raccolle de' pubblici Musei abbiano acquistata tale importanza da dar ragionevole fiducia che possano i loro visitatori trarne alimento di lodevole diletto e di istruzione, molto allora giova che se ne pubblichino i cataloghi sistematici per guida e stimolo ai medesimi. Sono i detti cataloghi di grande ajuto pell' acquisto di nuove cognizioni agli studiosi che facciansi a consultare i libri ove que' tali oggetti sono descritti col nome loro scientifico. E servono poi a notificare ai Naturalisti d' ogni paese lo stato delle raccolte che possono interessarli: in particolar modo graditi ed utili ai Direttori de' Musei, ed a' possessori di speciali raccolte, per l' agio che loro è fatto di procacciarsi novelli acquisti mediante compere o cambi, a norma delle circostanze. A questo scopo basta un indice sistematico degli oggetti colla indicazione della loro patria rispettiva.

Fra le raccolte dei Vertebrati possedute da questo Museo civico la più ricca di specie è quella dei Rettili ed Anfibi; perocchè ne racchiude più di due terzi di quelli rinvenuti finora viventi sulla terra.

Il Principe Carlo L. Bonaparte nel suo Conspectus Systematum Herpetologice et Amphibiologice stampato nell' anno 1850 ne annovera de' conosciuti sino allora 1200. Nell' opera che ha servito di base alla disposizione di questa nostra raccolta Erpétologic générale par A. M. C. Dumeril et G. Bibron. - 
Paris 1854-1834 IX Vol.: ove sono descritte lutte le specie note, comprese pure le Aggiunte indicate nel Répertoire des ordres, familles, genres et espèces de la classe des Reptiles, appendice al volume $\mathrm{IX}^{0}$ che si è dato in luce nell' anno 1854, il numero delle specie ascende in complesso a 1427.

Ora se si confronti con queste cifre quella che si rileva dal nostro Catalogo, apparirà che pochi e solamente i primari Musei possono vantare una più ricca, ed anche solo una simile Raccolta di Rettili ed Anfibi, o si guardi al numero o guardisi alla rarità delle specie. Anzi parecchi ve n' ha i quali ben provveduti nelle altre tre classi di Vertebrati, molto, proporzionatamente, difettano in questa, e forse la ragione sta nella difficoltà che s'incontra a farne gli acquisti, come ognuno può agevolmente comprendere $\left(^{1}\right)$.

Furono queste considerazioni che promossero la pubblicazione del presente Indice dei Rettili ed Anfibi. Io mi sono per lo più attenuto all' opera succitata di Duméril e Bibron,

(1) Non ispiacerà, penso, ch'io qui riferisca le cifre de' Rettili ed Anfibi portate dai Cataloghi stampati de' varj Musei che sono a mia cognizione. E così il Museo di

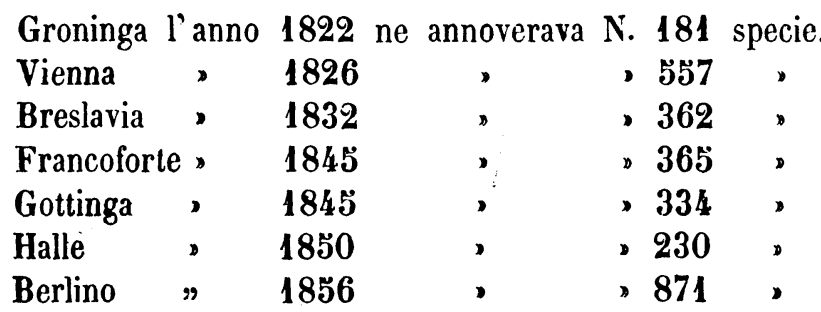

Tralascio l' indicazione della cifra delle specie de' Rettili ed Anfibi annoverate nel Catalogo del Museo di Londra e di Parigi per doppio motivo, primo perchè nè l' uno nè l'altro di questi Cataloghi è finora compito (( l'une di Londra non comprende che i Cheloni (venne pubblicato nel 1844, un'edizione in $4 .^{\circ}$ nel 1856), i Sauri (nell' anno 1845) qualche famiglia degli Ofidi (1849) e gli Urodeli (1850); e l'altro di Parigi che si pubblicò nell'anno 1851 contiene soltanto i Chẹloni e Sauri)) secondo perchè in questi, Cataloghi sono anche indicate molte specie mancanti ai suddetti Musei. 
e sebbene mi paresse opportuno qualche cambiamento nella disposizione delle specie in generi e famiglie degli Ofidi ed Anfibi, ho voluto qui, tranne poche deviazioni, astenermi da ogni innovazione, imperocchè proponendomi di pubblicare in apposita aggiunta la descrizione delle specie nuove dal $\mathbf{M u}$ seo possedute, avrò agio di esporre in quella occasione la particolare opinione mia a questo riguardo.

Debbo avvertire qui che ogni qualvolta nel presente Indice al nome della specie è anteposto un asterisco (ciò significa che il Museo ne possiede individui disponibili che si offrono in cambio d' altri delle specie mancanti alla raccolta, ed inoltre che se dopo il nome della specie quello dell' Autore è posto fra parentesi (-) è segno che mi è ignoto, se tale specie sia stata già descritla in qualche opera.

Se poi dopo il nome della specie, non è indicato l'autore che primo l' ha descritta, ciò denota che il nome dato non è che scelto provvisoriamente da me per non lassciare senza una sua indicazione la specie nella raccolta e potervela collocare a canto alle specie aflini. E dico scelto provvisoriamente, perchè sono ben alieno dal credere che siano tutte specie non ancora descritle. Chi conosce questo genere di studi converrà meco delle difficoltà che presentano talora le specie per l'inesattezza delle descrizioni e per la qualità dei dubbi che sorgono intorno la loro determinazione, dubbi che sovente non si possono sciogliere se non per l' autopsia dell' individuo medesimo che ha servito alla descrizione. D' altra parte molte di tali descrizioni sono inserite in opere periodiche, od altre che non sempre è facile di procurarsi.

Pertanto io non ho voluto arrischiare fin d' ora veruna de cisione su quelle specie le quali forse per l' insufficienza dei miei studi non seppi ritrovare già descritte. Ma spero che nell' appendice che mi propongo di fare al presente Indice, potrò riparare a varj di simili difetti, e allora solo conservare $i$ nomi delle specic veramente nuove, dandone in essa appen- 
dice la descrizione. Perchè solo la specie bene descritta entra nel patrimonio della scienza; i semplici nomi privi della indicazione dei caratteri distintivi della specie non essendo di alcun valore scientifico, ma, si, dannoso incremento della già intricata sinonimia, vera zavorra della quale conviene ormai alleggerire la scienza. E ciò è tanto più da ritenersi in quanto che nella storia naturale l' antico adagio - qui bene distinguit, bene docet - ̀̀ aurea regola, ̀̀ norma sicura: imperocchè la distinzion delle specie è la base sulla quale posano tutte le altre nozioni che emergono dallo studio zoologico, fisiologico ed anatomico della specie ritenuta e conosciuta da tutti i Naturalisti onde instituire l' esame e ripetere le osservazioni. $L$ ' aggruppamento delle specie in generi e famiglie è in gran parte dipendente dalle parziali vedute di chi se ne occupa, e sempre subordinato alla cognizione delle specie.

Prima di terminare questo già forse troppo lungo esordio mi sia permesso di accennare soltanto che degli Ofidi, sui quali ho fatto da varj anni uno studio particolare, ho già approntate le descrizioni degli individui d' ogni specie che trovasi nella nostra raccolta nella mira di renderle pubbliche poi, nella speranza che un tal lavoro contribuisca a diffonderne la conoscenza, e possa in qualche modo chiarire la distinzione delle specie assai oscura finora per coloro che non misero uno studio particolare in questo Ordine di rettili. E già chi di tale materia si è in special modo occupato consentirà meco che spesso, per la insufficienza delle descrizioni e per le pessime figure che talora vengono citate, si entra in un laberinto di dubbi in riguardo a certe specie dal quale è molto difficile l' uscire. Anzi nella persuasione in cui sono venuto coll' inoltrarmi in questi studi che talora anche le più accurate descrizioni non bastano alla ricognizion della specie, se non si ha innanzi qualche disegno esatto della medesima, era mio vivo desiderio di potere accompagnare le mie descrizioni, nella loro pubblicazione, con disegni che porgessero 
una immagine fedele d'ogni specie descritta; e tutti i particolari caratteristici della stessa necessari a conoscersi. Nel che ebbi la fortuna propizia, avendo io ritrovato nel giovine mio allievo il sig. Ferdinando Sordelli un animo invaghito della scienza, un occhio intelligente, una mano esperta in cosi fatti lavori: onde già mettendo a profitto di scopo scientifico queste belle qualità, dà ora opera ad eseguire sotto la mia direzione le desiderate figure con somma precisione e con tanto zelo ed assiduità che già ne sono ritratte per più di due terzi le specie della raccolta.

Non mi sarei indotto a far qui cenno di questa, comechè già ben progredita impresa prima che il lavoro fosse al punto da comparire in luce (lo che avverrà il più tosto che le circostanze lo permettano), se non mi vi avesse persuaso il desiderio di approfittare di questa presente occasione per indirizzare le mie preghiere ai Direttori di Musei zoologici ed ai possessori di raccolte di Ofidi onde vogliono graziosamente cedere quelle specie che per avventura avessero disponibili e che mancano a questa raccolta. E già sommo sarebbe il loro favore se anche tali specie di cui non volessero privarsi per cambio, avessero la squisita gentilezza di comunicarmi soltanto per la ispezione, le quali senza indugio e sottostando a qual si sia spesa io rimanderei: gratissimo a loro della cortese benevolenza colla quale avrebbero contribuito a portar a miglior termine e perfezione, e render più utile questo, qual ch' esso sia, nostro coscienzioso lavoro.

Milano, 15 Gennajo 1857.

Prof. G. JaN,

Direltore del Museo civico. 


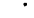




\title{
REPTILIA ED AMPHIBIA
}

\author{
MUSAEI MEDIOLANENSIS
}

\section{REPTIIIA}

TESTUDINATA

(Chelonia)

TERRESTRIA

(Chersobia)

Testudo L.

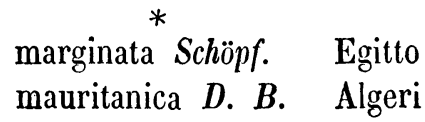
* *

graeca $\boldsymbol{L}$.

Toscana

geometrica $L$.

radiata Shaw.

Capo

tabulata Walb.

Madagascar

carbonaria Spix.

America mer.

polyphemus Daud. America sett.

elephantopus Harl. Gallopagos * * *

angulata Daud.

Homoeopus D. B.

areolatus Thunh. Africa austr.

Cinixys Bell.

erosa Gray.

Belliana Gray.

\section{Natal}

Gabon

Senegambia

\section{PALUSTRIA}

(Limnobia)

a. CRYPTODERA

Cistudo Flem.

carolina $\boldsymbol{L}$.

chrysopharia

America sett.

europaea Schöp

Lombardia

Blandingii Holbr. America sett.

Diardii $D . B$.

var. sumatrana Sumatra

Emis. D. $\boldsymbol{B}$.

caspica Schweigg. Soria

leprosa Schweigg. Spagna

(sigris D. B.)

punctularia Daud. America mer. pulchella Schweigg. \ geographica Lesueur. Amer. sett. pseudogeographica Les. D , concentrica Shaw. ग , oregonensis Harlan. \ serrata Daud. irrigata Bell. ornata Bell. America mer. guttata Schneid. picta Herm. cumberlandensis Holbr. 
$\begin{array}{ll}\text { trijuga Schweigg. } & \text { Giava } \\ \text { borneoensis (Schleg.) } & \text { Bornco } \\ \text { tectum Bell. } & \text { Gange } \\ \text { crassicollis Bell. } & \text { Sumatra }\end{array}$

Chelydra Schweigg.

(Emisaurus D. B.)

serpentina $\boldsymbol{L}$.

Staurotypus Wagl.

triporcatus Wagl. Messico

odoratus Latr.

Cinosternon Wagl.

America sett.

scorpioides $\boldsymbol{L}$.

Messico

Surinam

pensylvanicum Gmel. America sett. leucostomum $D$. B. Nuova Orlean.

\section{b. Pleurodera}

Podocnemis Wagl.

expansa Schweigg. America mer.

unifilis Trosch.

Pelomedusa Wagl.

(Pentonyx Rüpp.)

galeata Schöpf.

Capo

(capensis D. B.)

gehafiae Riipp. Abissinia

Sternotherus Bell.

Derbyanus Gray. Madagascar

Platemys Wagl.

planiceps Sclneid. Brasile

(martinella Daud.)

Neuwiedii D. B. Brasile

Chelodina Fitz.

flavilabris $D . B$. Brasile

Maximiliani Mik. Brasile

Chelys Schweigg.

fimbriata Schneid. Brasile (matamata Brug.)

\section{FLUVIATILIA}

(Potamobia).

Tryonyx Geoffr.

(Gymnopus D. B.) ferox penn

America sett.

(spiniferus Les.)

muticus Les.

America sett.

aegyptiacus Geoffr. Egitto

ocellatus Hardw. Gange

javanicus Geoffr. Giava

japonicus Schleg. Giappone

subplanus Geoffr. Gange

sinensis Wiegm. China

Cryptopus $D . B$.

(Emyda Gray).

granosus Schöpf. Pondichery

MARINA.

(Thalassobia).

Chelonia Brongn.

midas Lin.

Oceano atlan.

maculosa Cuv.

Coste del Malabar.

imbricata $L . \quad$ Oceano atlan.

caouana Lacép. Mediterraneo

olivacea Esch. Singapore

(Dussumieri D. B.)

Sphargis Merr.

coriacea $\boldsymbol{L}$.

Oceano atlan.

\section{SAURIA}

\section{A S P I D O T A}

\section{CROCODILI}

Alligator Cuv.

palpebrosus Cuv. America mer. lucius Cuv. America sett.

sclerops Schn. America mer.

punctulatus Spix. Brasile

Crocodilus Cuv.

vulgaris Cuv. Egitto

var. indica Schleq. India 
byporcatus Cuv. Gange acutus Geoffr. Martinica Gavialis Geoffr. gangeticus Geoffr. Gange

\section{LEPIDOTA}

\section{CHAMAELEONES}

Chamaeleo Laur.

vulgaris Cuv.

tigris Cuv.

pumilus Latr.

lateralis Gray.

senegalensis Cuv.

natalensis (Pöpp)

gracilis Hallow.

dilepis Leach.

pardalis Cuv.
Egitto, Algeri,

Portogallo

Seychelle

Capo

Madagascar

Ashantee

Natal

Gabon

Madagascar

\section{ASCALAB0TAE}

Platydactylus Cuv.

ocellatus Oppel. Madagascar

Cepedianus Cuv. Capo

var. madagascariensis. Madagascar

rapicauda Houtt. Antille

(theconyx $D . B$.)

pacificus Gray.

mauritanicus $L$. Genova, Nizza

(muralis D. B.) Dalmazia,Por-

aegyptiacus Ciuv. Cairo tog., Egitto

Delalandii $D . B$. Manilla

guttatus Daud. vittatus Houtt. monorchis Schleg. japonicus Schleg. Giappone homalocephalus Crevelds. Grava

Hemidactylus Cuv.

Peronii D. B.

Is. di Francia

variegatus $D$. B. Is. di Francia mutilatus Wiegm. ,

Hemidactylus.

\begin{tabular}{ll} 
servus (Boie) & Giava \\
maculatus D. B. & Malabar \\
verruculatus Cuv. & Dalmazia, \\
& Cipro, Egitto \\
mabouia Cuv. & Antille \\
var. Nattereri (Fitz) & Brasile \\
var. armatus Neuw. & \multicolumn{1}{c}{} \\
frenatus Schleg. & Giava, Timor \\
marginatus Cuv. & Giava \\
puellus (Schleg) & Ashantee \\
dactylus Cuv. & \\
Hasselquistii Schneid & Cairo, Biscara \\
fimbriatus Cuv. & Madagascar
\end{tabular}

Phyllodactylus Gray.

Lesueurii $D . B$. Nuova Olanda

porphyreus $D$. B. Capo

europaeus Gené. Sardegna

Sphaeriodactylus Cuv.

sputator Cuv. Cuba

punctatissimus D. B. S. Domingo

Gymnodactylus Spix.

elegans Gray. Nuova Zelan.

fasciatus $D . B$. India orient.

scaber Rüpp. Cipro, Candia

phyllurus Schneid. Nuova Olanda

Miliusii Bory.

Coleonyx Gray.

elegans Gray. Nuova Olanda

(Gymnod. coleonyx $A$. Dum.)

Stenodactylus Fitz.

guttatus Cuv. Egitto

\section{VARANI}

Varanus Merr.

arenarius Geoffr. Egitto

timoriensis Gray. Tímor

punctatus Gray. Tasmania

niloticus Hasselq. Egitto 
bengalensis Daud. Pondichery nebulatus Schleg. India orient. Picquotii. D. B. var. borneosensis Borneo bivittatus Kuhl. Giava chlorostigma Cuv. Nuova Zelan. varius Shaw. Nuova Olanda albigularis Daud. Capo ocellatus Rüpp. Abissinia ornatus. Costa d' oro

\section{IGUANAE \\ $a$. PLEURODONTA}

Polychrus Cuv.

* marmoratus $L$ Brasile anomalus Wiegm. ,

Laemanctus Wiegm. longipes Wiegm. Messico undulatus Wiegm. " Urostrophus $D$. $B$. Vautierii $D . B$ Norops Wagl.

Brasile

auratus Wagl.

Brasile, Mess. Anolis Daud.

Nitzschii Wiegm. Brasile (chrysolepis D. B.)

$\begin{array}{ll}\text { * * } & \\ \text { punctatus Daud. } & \text { Brasile } \\ \text { bullaris L. } & \text { Carolina }\end{array}$

(chlorocyaneus D. B.)

gracilis Neuw. Brasile nasicus $D . B$.

carolinensis Cuv. Georgia, Cuba (principalis L2)

nebulosus Wiegm. Messico alligator D. B. Manilla marmoratus $D . B$. America mer. cristatellus Gray. S.Lucia, Gua-

Sagraei Coct. Cuba Leachii $\boldsymbol{D}$. B. Guadalupa equestris Merr. Cuba heterodermus A. Dum. Nuova Gren. Ricordii $D . B$. S. Domingo *** chamaeleonides $\boldsymbol{D}$. B. Cuba Basiliscus Laur. mitratus Daud. Cayenne vittatus Wiegm. Messico Iguana. Laur.

- tuberculata Laur. America mer. rhinolopha Wiegm. Messico. nudicollis Cuv. Martinica Hernandesii. Messico Cyclura Harl. pectinata Wiegm. Messico acanthura Gray.

Brachylophus Cuv. fasciatus Cuv. Indie orient. Enyalus Wagl. rhombifer Spix. Brasile bilineatus $D$. B. Bahia Ophryoessa Boie.

superciliosa Lin. Brasile

Leiosaurus $D . B$.

Bellii $\boldsymbol{D}$. B. Messico

Hyperanodon $D . B$. ochrocollare Spix. Surinam Hypsibatus Wagl. agamoides Wiegm. Guiana punctatus Gray. Puerto $\mathrm{Ca}$ -

Centrura Bell. flagellifer Bell. Chili Holotropis $D$. B.

microlophus Coct. Cuba trachycephalus A.Dum. Nuova Grenada

Proctotretus D. B. chilensis Bell. Chili mosaicus Homb. et Jacq. " pictus Bell. tenuis $D$. B. nigromaculatus Wiegm. " Wiegmanni $D$. B. , Nattereri (Fits). Brasile 
signifer $D . B$.

Holbrookia Girard.

maculata Gir.

Cheroches

(Tennesée )

Tropidolepis Cuv.

$\begin{array}{lc}\text { undulatus Cuv. } & \text { Georgia } \\ \text { torquatus Wiegm. } & \text { Messico } \\ \text { formosus Wiegm. } & \triangleright \\ \text { spinosus Wiegm. } & \text { ॰ }\end{array}$

grammicus Wiegm. America sett.

variabilis ${ }^{*}$ Wiegm. Messico

microlepidotus Wiegm. ,

scalaris Wiegm.

Phrynosoma Wiegm.

Harlani Wiegm.

coronatum Blainv.

America mer., Texas

orbiculare Wiegm. Messico

Microlophus $D$. $B$.

peruvianus Less.

(Lessonii D. B.)

Ecphymotes Cuv.

torquatus Neuw. Brasile

Cherdaleus.

collaris.

Quito

Strobilurus Wiegm. torquatus Wiegm. Brasile Oplurus Cuv.

Sebae D. $B$.

Brasile

Doryphorus Cuv.

azureus Cuv.

Surinam

IIoplocercus Fitz.

spinosus Fitz. Brasile

\section{b. ACRODONTA}

Istiurus Cuv.

amboinensis. Cuv. Amboina

Lesueurii $D . B$. Nuova Olanda

Calotes Cuv.

cristatellus Kuhl. Amboina

jubatus $D . B$.

Giava timpanistriga Gray. Giava

ophiomachus Merr. Ceylon

versicolor Daud. Bengala, Calcutta

Lophyrus Merr.

tigrinus Merr. Amboina

minor (Schleg). Giava

Lyriocephalus Merr.

margaritaceus Merr. Indie orient.

Ceratophora Gray.

Stoddaertii Gray. Ceylon

Sitana Cuv.

ponticeriana Cuv. Indie orient.

Draco Lin.

fimbriatus Kuhl. Giava

Daudini $D . B$.

、 timoriensis Péron. Timor

lineatus Daud. Amboina

Grammatophora Kaup.

scalaris $D . B$.

Nuova Olanda

ornata Gray.

muricata Kaup.

barbata Kaup.

Agama Daud.

colonorum Daud. Senegal

nupta De Fil. Persepoli

* atra Daud. Capo

agilis Oliv. Egitto

sanguinolenta Eichw. Soria

aculeata Merr. Capo

hispida $\boldsymbol{L}$.

(spinosa D. B.)

mutabilis Merr. Egitto

sinaita Heyd.

Phrynocephalus Kaup.

Olivieri D. B. Persia

helioscopus Kaup. Siberia mer.

Stellio Daud.

vulgaris Daud. Egitto

caucasicus Eichw. Caucaso

Uromastix Merr.

spinipes Merr. Egitto

acanthinurus Bell. Algeri

Hardwickii Gray. Indie orient. 
Moloch Gray.

horridus Gray. Nuova Olanda

\section{LACERTINA.}

$a$. PLEODONTA

Crocodilurus Cuv.

lacertinus Daud. Brasile

Callopistes Grav.

(Aperomera D. B.)

coelestis D' Orb. Chili

(ornata D. B.)

Podinema Wagl.

(Salvator D. B.)

* Teguixin $L$.

Brasile

(Merianae D. B)

nigropunctatum Spix. Brasile

Ameiva Cuv.

vulgaris Licht.

major $D . B$.

Brasile, Venezuela

Cnemidophorus Wagl.

murinus Laur. Guiana

lemniscatus Daud. Guayra

* sexlineatus $\boldsymbol{L}$. Georgia

var. guttatus Wiegm Louisiana

Acranthus Wagl. viridis Merr. Brasile

Centropyx Spix.

calcaratus Spix. Guiana

\section{b. COELODONTA}

Tachydromus. Daud.

sexlineatus Daud. Borneo

japonicus D. B. Giappone

(Lac. tachydromoides Schleg.)

Tropidosaura Boie. algira $\boldsymbol{L}$.

Algeri

Lacerta $L$.

Algeri

nigropunctata

D. B. Fiume, Dal-
Fitzingeri Wiegm. Sardegna

agilis $\boldsymbol{L}$.

(stirpium Daud.) Lombardia

Lacerta.

viridis Daud. Lombardia e Dalmazia

var. strigata Eichw.

vivipara Jacq. B Brgamo

ocellata Daud. Nizza, Porto-

taurica Pall. Morea

muralis. Latr. Lombardia, Veneto, Dalm.

var. tiliquerta Cetti. Zara

* olivacea Rafin. ,

- cypria. Cipro

oxycephala Schleg. Dalmazia

Dugesii Milne Edw. Madera

perspicillata $D$. B. Algeri

Psammodromus Fitz.

Edwardsii D. B. Francia mer.

Ophiops Ménétr.

elegans Ménét. Cipro

Acanthodactylus Fitz.

vulgaris $D . B$ Algeri

capensis Smith. Capo

scutellatus Aud. Egitto

Savignyi Aud. Cipro

Boskianus Daud. Egitto

Scapteira Fitz.

grammica Licht. Tartaria

Eremias Fitz.

variabilis Pall. Crimea

rubropunctata Licht. Egitto

pardalis D. B. Arabia, Egitto, Dalmazia

\section{CYCLOSAURA}

a. PTYCHOPLEURA

Zonurus Merr.

cordylus $\boldsymbol{L} . \quad$ Capo

(griseus Cuv.) 
Lepidophymus A. Dum.

flavomaculatus A. Dum. Messico

Gerrhosaurus Wiegm.

flavogularis Wiegm. Mozambico

Proctoporus Tschudi.

pachyurus Tschud. Nuova Gran.

Osculatii. Quito

Espondylus Tschud.

maculatus Tsch. Peru

Gerrhonotus Wiegm.

Deppii Wiegm. Messico.

taeniatus Wiegm. ,

imbricatus Wiegm. ,

licheniger Wagl. ,

Pseudopus Merr.

Pallasii Opp.

Ophisaurus Daud.

* ventralis Daud. Georgia.

var. $a-c$.

Chamaesaura Schneid. anguina $\boldsymbol{L}$.

Chalcides Daud.

Cuvieri Wagl. Colombia.

cophias Merr. Cayenne.

Schlegeli D. B. ,

\section{$b$. GLYPTODERMA}

Trogonophis Kaup.

Wiegmanni Kaup.

Amphisbaena $\boldsymbol{L}$.

fuliginosa $L$. Brasile.

alba $\boldsymbol{L}$.

var. flavescens Wagl. ,

vermicularis Wagl.

Darwinii D. B. Patagonia.

Kingii Bell. Buenos-Ayres.

cinerea Vandelli. Cipro.',

Lepidosternon Wagl.

microcephalum Wagl. Brasile.

scutigerum Hempr.

\section{SCINCOIDEA}

$a$. SAUROPHTHALMA

Scincus Laur.

officinalis Laur. Egitto.

Sphenops Wagl.

capistratus Wagl. Egitto.

Diploglossus Wiegm.

occiduus Shaw. Giamaica.

(Shawi D. B.)

Houttuynii Coct. Brasile.

Pleii $D . B . \quad$ Messico.

Gongylus Wiegm.

ocellatus Wagl. Egitto, Cipro.

Boierii D. B. Is. Bourbon.

Copeoglossum Tschud.

cinctum Tschud. Perù, Quito

Eumeces Wiegm.

punctatus Wiegm. Bengala, Manilla]

agilis Raddi. Brasile

(Spixii D. B.)

mabouia Coct. Martinica

Lessonii $D . B$ Tongatabo, Amboina

spec.

Capo

Euprepes Wagl.

Perrotetii D. B. Senegal, Costa d'oro

striatus Halow. Gabon

* Merremii D. B. Capo vittatus Oliv. $\quad$ Egitto

(Olivieri D. B.)

quinquetaeniatus Licht. Egitto

(Savignyi Aud).

var. $a-c$.

punctatissimus Smith. Capo

septemtaeniatus Reuss. Abissinia

Delalandii $D . B$. Capo verde

rufescens Shaw. Bengala

(Sebae D. B.) 
Plestiodon $D . B$. auratum Schneid. Algeri

(Aldrovandi D. B.)

laticeps Schneid. Georgia

quinquelineatum $L$. "

Lygosoma Gray.

Guichenoti. Gray. Nuova Olanda hieroglyphicum Homb el Jacq.

Duperreyi. D. B. "

moco $D$. $B$.

Nuova Zelan.

laterale Hollor. America sett.

brachypus Schneid. Giava

(brachypodum D. B.)

melanopogon $D$. B. Timor

Temminckii $D . B$. Molucche

Quoyi. D. B.

Nuova Olanda

Labillardieri Coct. \,

taeniolatum Shaw. \,

moniligerum Valenc. „

smaragdinum Less. Amboina

Leiolepisma $\boldsymbol{D}$. $\boldsymbol{B}$.

Telfairii Coct.

Is. Gabriel

Tropidolepisma $D . B$.

Dumerilii $D . B$. Nuova Olanda

Cunninghami Gray. " "

Cyclodus Wagl.

casuarinae D. B. Nuova Olanda

nigro-luteus Wagl. » »

gigas Bodd.

(Boddaertii D. B.)

Trachysaurus Gray. rugosus Gray. Nuova Oland.

Heteropus Fitz.

fuscus $D . B$.

var. bilineata.

Is. di Waigiou

Tetradactylus $P$ er.

decresiensis Per. Isola Decrés

(Nuova Oland.)

Hemiergus Wagl.

decresiensis Per. Nuova Oland.

Seps Daud.

chalcides $L$.

Anisoterma A. Dum. sphenopsiforme A. Dum. Senegal.

Heteromeles $\boldsymbol{D}$. $\boldsymbol{B}$.

mauritanicus Guichen. Algeri.

Heteropodium (Fitz.)

Kotschyanum (Fitz.) Caucaso.

Scelotes Fitz.

bipes $\boldsymbol{L}$.

Capo.

(Linnaei D. B.)

Praepeditus $D$. B.

lineatus $D . B$. Nuova Oland.

Anguis $\boldsymbol{L}$.

fragilis $L$. Lombardia.

Ophiomorus D. B. problematicus.

Acontias Cuv.

meleagris Cuv. Capo.

nigra Peters. Mozambico

\section{b. OPHIOPHTHALMA}

Ablepharus Fitz.

pannonicus Schreib. Ung., Cipro

(Kitaibeli Coct.)

plagiocephalus Per. Nuova Oland.

(Peronii D. B.)

lineoocellatus Gray.

Gymnophthalmus Merr.

quadrilineatus Lin. Martinica.

Pygopus Merr. (Hysteropus D. B.)

lepidopus Merr. Nuova Oland.

(H. Novae-Hollandiae D. B.)

Lialis Gray.

punctulata Gray. Nuova Oland.

Dibamus $D . B$.

celebensis (Schleg.) Celebes.

Typhline Wiegm.

aurantiaca Peters. Mozambico 
OPHIDIA

\section{AGLYPHODONTA}

\section{TYPHLINA}

(Epanodonta D. B.)

Pilidion D. B.

lineatum Reinw. Giava Ophtalmidion $D . B$.

mossambicus

Mozambico

Eschrichtii Schleg. Cos.diGuinea Onychocephalus. D. B.

Delalandii Schleg. Capo

Typhlops Schneid.

reticulatus Lin. Bras. Caracas

lumbricalis Lin. Guadalupa

Richardii D. B. Antille

vermicularis Merr. Cipro

braminus Cuv. Pondichery

Cephalolepis $\boldsymbol{D}$. $\boldsymbol{B}$.

squamosus Schleg. Guyana

(leucocephalus D. B.)

Anomolepis

mexicanus.

Messico

(Catodonta D. B.)

Stenostoma.

albifrons Wayl. Brasile

\section{LROPELTA}

Coloburus D. B.

ceylanicus Cuv. Ceylon

Plectrurus.

Perrotetii D. B. Mont. Nilgerrhy (Ind. or.)

\section{TORTRICINA}

Tortrix.

scytale Oppel. Guyana

Xenopeltis Wagl.

unicolor Wagl. Giava
Cylindrophis Wagl.

melanotus.

Celebes

rufus.

Giava

maculata.

Ceylon

\section{ACROCHORDINA}

Chersydrus Cuv.

granulatus Schneid. Sumatra

(fasciatus Shaw.)

Xenoderma Reinh.

javanicum Reinl. Giava

f) ERYCINA

Eryx Daud.

Johnii Russ. Dekan

jaculus Daud. Cairo

thebaicus Geoffr. Egitto

conicus Schneid. Malabar

(6 PYTHONINA

(Holodonta D. B.)

Morelia.

argus Klein. Nuova Olanda

Python. Daud.

Sebae D. B. Africa occid.

regius $D . B . \quad$,

molurus $L$ Bengala

hieroglyphicus Costa d' oro

reticulatus Schneid. Sumatra

Liasis Gray.

amethystinus. Amboina

(Aprolerodonta D. B.)

Xiphosoma Wagl.

caninum $\boldsymbol{L}$. Cayenne

hortulanum $L$. Brasile

madagascariense $\boldsymbol{D}$. $\boldsymbol{B}$. Madagascar

Epicrates Wagl.

cenchris $L$ Brasile

angulifer $D . B . \quad$ Cuba 
Epicarsius Fisch.

cupreus Fisch. Puerto Cabel.

Enygrus Wagl.

carinatus Schneid. Guinea

Bibronii Hombr.et Jacq. Nuov. Oland.

Leptoboa $\boldsymbol{D}$. $\boldsymbol{B}$.

Dussumieri Schleg. S. Maurizio

Tropidophis $D$. B.

melanurus Schleg.

S. Lucia

maculatus $D . B$. Cuba

Boa. Lin.

constrictor Lin. Brasile

diviniloquax Schn. Portoricco

imperator Daud. Cordova

(Messico)

Eunectes Wagl.

murinus $\boldsymbol{L}$.

Cayenne

Chilabothrus $D$. B.

inornatus Reinh. Portoricco

\section{CALAMARINA}

Oligodon Boie.

bitorquatum Boie. Giava

(subquadratum D.B.)

sublineatum D. B. Batavia

subgriseum D. B. Pondichery

Calamaria Boie.

Linnei Boie.

var. tessellata Boie. Giava

punctatolineata.

vermiformis. D. B. Giava

Rhabdosoma $D . B$.

semidoliatum $D . B$. Messico

badium Boie. Surinam

torquatum Boie. $\quad$ D

subtorquatum. Brasile

crassicaudatum D. B. Nuova Gran.

fuscum.

Messico

Homalosoma Wagl.

lutrix Lin.

Capo

coronella Srhleg. Morea
Scolecophis (Filz.)

cingulata (Fitz.)

Eucritus

atrocephalus. Mozambico

Rhabdion $D$. B.

Forsteni. D. B. Celebes

Elapoides Boie.

fuscus Boie.

Sumatra

Aspidura Wagl.

scytale Wagl.

Ceylon

Celuta B. G. (Carphophis D. B.)

amoena $S a y$.

Louisiana,

Tennessee

Osceola $B . G$.

elapsoidea Holbr. Georgia

Haldea $B$. $G$.

(Conocephalus D. B.)

striatula Lin.

Nuov.Orlean.

Dochis

strigata Venezuela

\section{COLUBRINA}

(Coryphodonta D. B.)

Coluber Lin. (Dum. Prodi, d. Ophid.)

(Coryphodon D. B. Erp. Gen.)

pantherinus Merr. Brasile, Surinam

constrictor Lin. America sett. Messico

Blumenbachii Merr. Bengala

korros Reinw. Sumatra,

Malacca

mentovarius D. B. Messico

Plesius

inconstans. Messico

(Syncrantheria D. B.)

Coronella Laur.

laevis Laur. Lombardia

girondica Daud. Toulon

(Riccioli. Metaxa) Roma 
var.? dalmatina. Zara

cana Lin. juv. margaritacea Merr. ,

$\begin{array}{ll}\text { getulus Lin. } & \text { Georgia } \\ \text { Sayi Dek. } & \text { Nuova Orlea. } \\ \text { doliata Lin. } & \\ \text { formosa Schleg. } & \text { Messico } \\ \text { spec. } & \text { Caucaso } \\ \text { spec. } & \text { Messico }\end{array}$

Simotes D. B.

Russelii Dard. Manilla octolineatus Schneid. Ind. orientale coccineus Blumenb. America sett. pictus.

spec.

Tropidonotus Kuhl.

\begin{tabular}{|c|c|}
\hline $\begin{array}{l}\text { natrix } \boldsymbol{L} . \\
\text { var. bilineala. } \\
\text {, subbilineata. } \\
\text {, nigra. } \\
\text { collaris. } \\
\text { tessellatus Laur. } \\
\text { (viperinus Latr.) } \\
\text { chersoides Wagl. } \\
\text { hydrus Pall. } \\
\text { chinensis. }\end{array}$ & $\begin{array}{l}\text { Lomb. Veron. } \\
\text { Dalmazia } \\
\text { ' } \\
\text { Messico } \\
\text { Lomb. Veron. } \\
\text { Dalmazia } \\
\text { Sicilia, Egitto } \\
\text { Troade } \\
\text { China }\end{array}$ \\
\hline
\end{tabular}

fasciatus Lin. America sett.

var. sepedon Holbr. Georgia " niger Holbr. pogonias $D$. B. Messico leberis Lin. catenulatus.

sirtalis Lin.

Ceylon

Giava

Lomb. Veron.

Dendrophis Boie.

pictus Boie

var.polychrous Schleg. Sumatra

formosus (Schleg.) Giava

melanostigma. Mozambico

inornatus.

Africa occid.

albovariegatus(Smith.) Africa merid.

octolineatus $D$. B. China

Chlorosoma Wagl.

vernalis Holbr.

Texas

Hapsidrophis Fisch.

nigrolineatus Schleg. Ashantée

Herpetodryas Boie

carinatus $\boldsymbol{L}$.

Brasile, Demerrara

var. a - i.

var. fuscus $L$. Brasile

Poiteaui D. B. Cayenne

aestivus Lin. America sett.

(bipunctatus Schleg)Messico

var. ordinatus $L$. America sett.

saurita Lin.

seychellensis Schleg. Seychelles

trianguligerus Schleg. Giava

quincunciatus Schleg.

var. A et C (D. B.) Giava

vibakari Boie.

Giappone

schistosus Daud. Pondichery

spilogaster Boie. Manilla

vittatus Lin.

Giava flagelliformis Catesb. Charlestown

Boddaertii Seetz. Cayen., Mess.

xanthogaster (Fitz) Brasile

Bernieri D. B. Madagascar

Spilotes Wagl.

variabilis Neuw. Brasile

poecilostoma Nenw. ,

corais Cuv.

chalybaeus.

Surinam, Br.

Surinam 
melanurus D. B. Messico

Rhinechis Michahell. scalaris Schinz.

Rhinocleis gradatus.

Pituophis Holbr. melanoleucus Daud. California mexicanus $D . B$. Messico

Elaphis D. B.

quadriradiatus Gmel. Roma, Zara virgatus Schleg. Giappone var. atra.

Pöppigi. Georgia

alleghanensis Holbr. Texas guttatus $\boldsymbol{L}$. America sett. flavescens Scop. Lomb. Dalm. (Aesculapii Host.) conspicillatus Boie. Giappone Campsosoma D. B. radiatus Schleg. Giava subradiatus Schleg. Timor melanurus Schleg. Giava

Ablabes D. B.

rufulus Licht.

punctatus Lin.

var. impunctala.

balioderus Boie.

Capo

California America sett. eximius De Kay. America sett. (triangulum Lacép?)

quadrilineatus Pall. Dalmazia

(Calopeltis leopardina Bonap)

Enicognathus $D$. $B$.

melanocephalus Lin. Brasile rhodogaster Schleg. Madagascar * * * *

Calopisma D. B.

erythrogrammus $\boldsymbol{P a l}$. $d$. Beauv.

abacurus Holbr. America sett. plicatilis $\boldsymbol{L}$. Louisiana Brasile Tretanorhinos $D . B$. mexicanus. Messico

\section{DIACRANTHERA}

Helicops Wagl. carinicaudus Neuw. Brasile Wagleri. America cent. angulatus Lin. Brasile gastrostictus (Fitz) , Leprieurii $D$. $B$.

Xenodon Boie.

severus $\boldsymbol{L}$.

rhabdocephalus Neuw. Treuensteini (Mus. Vindob.), Nattereri (Fitz.) typhlus Schleg. Cayenne gigas $D$. $B$. Brasile

Heterodon Latr.

platyrhinos Latr. Georgia var. nigrescens. Tennessee niger (Catesb) Troosl. America setl. madagascariensis $D . B$. Madagascar * *

Dromicus D. B.

cursor Shaw. Antille

lineatus Lin.

Surinam Brasile, Messico

unicolor D. B. Antille infrenatus. Brasile antillensis Schleg. Antille Temminckii Schleg. Chili leucomelas D. B. Guadalupa angulifer $D . B$. Cuba S. Vinc. (Antille)

spec.

Caracas

Etairejus rhodorachis. Persia

Periops. Wagl.

hippocrepis Lin. Italia merid. var.? algira. Algeri

cypria. Cipro

- parallelus Wagl. Cairo

Pseudoperiops persicus. Schiras

Zamenis Wagl. 
viridiflavus Lacép. Milano, Par. Scydropus

var. carbonarius. Dalmazia

- jugularis. Cipro

trabalis Pall.

(caspius Lepech.) Angora, Odes.

florulentus Schleg. Egitto

Dahlii Fitz. Spalat. (Dal.)

spec.

Liophis Wagl.

cobella $L$.

reginae $L$.

ocellatus.

cinctus.

Egitto

bicinctus Herm.

var. A. B.

bivittatus.

viridis.

Merremii Neuw.

Brasile

Bra. Surinam

Brasile

California

Caracas

Brasile

var. poecilogyrus Neuw. Brasile

v. Forsteri Spix. Surinam

leucomelas Trosch. Caracas

Amphiesma $D$. B.

$\begin{array}{ll}\text { stolatum L. } & \text { Ceylon } \\ \text { tigrinum Boie. } & \text { Giappone } \\ \text { subminiatum Reinw. Giava } \\ \text { rhodomelas Schleg. } & \text { Borneo } \\ \text { chrysargos Boie. } & \text { Giava }\end{array}$

\section{LEPTOGNATHA.}

Petalognathus $\boldsymbol{D}$. B. nebulosus $L$.

Surinam

Anholodon $D$. $B$.

Mikani (Mus. Vind.) D. B. Brasile

Dipsadomorus $D$. $B$.

indicus $L$ aur.

Leptognathus $\boldsymbol{D} . \boldsymbol{B}$.

pavoninus $\mathrm{Cuv}$.

Cochliophagus $D$. B.

Sumatra

inaequifasciatus $D . B$. Brasile

Hydrops Wagl.

Martii Wagl.

Brasile

var. ornatissimus (Fitz.) , mexicanus.

Rachiodon $D . B$.

scaber $\boldsymbol{L}$.

ntée ,

Cairo

Storeria B. G.

(Ischnognathus D. B.)

Dekayi Holb.

Georgia, Mes.

occipitomaculata B. G. America sett.

Nattereri.

Brasile

Tropidopeltis (Fitz.)

strigiventris (Mus. Vind.) Brasile

Brachyorrhos Kuhl.

albus $\boldsymbol{L}$.

Streptophorus D. B.

Sebae $D . B$.

Giava

remmatognathus $D . B$.

Catesbyi Weigel. Brasile

Schlegelii.

Caracas

\section{LYGODONTA.}

Boaedon D. B.

lineatum $D . B . \quad$ Costa d' Oro

capense Smith. Capo, S. Tomaso

dubium. Gabon

Eugnathus.

geometricus Boie. Capo

Lycodon Boie.

aulicum $L$. Bengala

var. $a-d$.

Giava

lividum Sal. Miull. Is. Pulo Samao (Ind. or.)

spec.

China

Cyclochorus D. B.

lineatus Reinh. Manilla

Ophites Wagl.

subcinctus Wagl. Giava * *

Lamprophis Fitz.

aurora $L$.
Capo 
Pareas Wagl.

carinatus Reinw. Giava Amblycephalus Boie.

(Aplopeltura D. B.)

boa Boie.

Mollucche

Odontomus $D$. B.

nympha Daud. Bengala

\section{GLYPHODONT A}

Innoxia

\section{OPISTOGLYPHA}

\section{ANISODONTA}

(et Oxycephala D. B. in parle).

Langaha Brug.

madagascariensis Lacép. Madagascar (ensifera $D . B$ )

Dryophis Schleg.

nasutus Merr.

(Dryinus nasutus D. B.

prasinus Reinw. Indie orient.

Tragops prasinus. Wagl. var. xanthozonius Kuhl. Giava

Kirtlandi Hallow. Gabon

(Oxybelis Kirtlandi $D . B$.)

Bucephalus Smith.

typus Smith.

Hemiodontus. D. B.

Capo

leucobolia Schleg. Ceram, (Mo-

Psammophis Boie. lucche)

$\begin{array}{ll}\begin{array}{c}\text { sibilans } \boldsymbol{L} . \\ \text { (moniliger Daud.) }\end{array} & \text { Egitto } \\ \text { crucifer Merr. } & \text { Capo } \\ \text { amoena. } & \\ \text { irregularis Fisch. } & \text { Cost. di Guin. } \\ \text { elegans Shaw. } & \text { Capo } \\ \text { pulverulenta Boie. } & \text { Giava } \\ \text { Perrotetii } D . B . & \text { Dekan }\end{array}$

Opetiodon D. B.

cynodon $\mathrm{Cuv}$. Cost. di Guin.

Tarbophis Heischm.

vivax Fitz. Dalmazia

Lycognathus $D . B$.

scolopax Klein Bolivia

(Lyc. audax Schleg.)

leucocephalus Mik. Brasile

cucullatus Geoffr. Algeri

Tomodon D. B.

lineatum D. B. Messico

spec. Tampico

\section{STENOCEPHALA.}

Elapomorphus Wiegm.

lemniscatus D. B. Santa Cruz

Erythrolamprus Boie.

Aesculapii $\boldsymbol{L}$. Brasile

larvatus Messico

venustissimus Neuw. Brasile

var. $B$.

Messico

Homalocranion $D . B$.

melanocephalum $\boldsymbol{L}$. America sett.

Stenorrhina

Freminvillei D. B. Messico

varians

var. $A-C$.

De Filippii.

Messico

\section{SCYTALINA.}

Rhinosimus $D$. B.

Guerinii $D . B \quad$ Puerto Cabel.

Rhinostoma Fitz.

nasuum Wagl. Puerto Cabel.

proboscideum (Fitz.) Brasile

Scytale Boie

coronata Schneid. ,

Neuwiedii $D$. $B$.

modesta

spec.

America cent.

Bahia 
Brachyrrhyton $D$. B. plumbeum Neuw. Brasile cloelia Daud.

Oxyrrhopus Wagl.

a trigeminus D. B. Bahia, Brasile doliatus D. B. Tampico formosus Neuw. Brasile clathrathus $D . B$, immaculatus $D$. B. , bipraeocularis $D . B$. ग

petolarius $\boldsymbol{L}$.

America mer.

Chrysopelea Boie. ornata Shaw.

Giava

Oligotropis Reinv. rhodopleura Reinw. Amboina

\section{PLATYRHINA.}

Hypsirrhina Wagl.

enhydris Schneid. Giava

maculata $D . B$. China

Eurostus D. B.

plumbeus Boie. Giava

Homalopsis Kuhl.

buccata $\boldsymbol{L}$.

Giava

Cerberus Cuv.

boaeformis Schneid. Giava

\section{DIPSADINA.}

Telescopus Wagl.

obtusus Reuss. Egitto

Rhinobothrium Wagl. lentiginosum Scop. Guyana

Himantodes $D$. B. cenchoa Lin. Caracas

Triglyphodon $D$. B.

dendrophilum Reinw. Giava

gemmicinctum $\boldsymbol{D}$. B. Celebes

Dryophylax Wagl.

viridissimus Lin.

Olfersii Licht.

America mer.

serra Schleg.

Scholtii Fitz.
Coelopeltis Wagl.

lacertina Wagl. Dalmazia, Algeri

(insignita Geoffr.)

0xybelis $W a g l$.

argenteus Daud. Brasile

Catesbyi Schleg. Surinam

aeneus Wagl. Brasile

Dipsas Boie.

trigonata Schneid. Bengal.Dekan

Scherzeri (Fitz.) Costaricca

multimaculata Reinw. Giava

annulata Lin. Messico, Surinam

colubrina Schleg. Madagascar

pulverulenta Fisch. Africa occid.

Nattereri Mik. Brasile

punctatissima Schleg. Cayenne, Brasile

$\begin{array}{ll}\text { rhombeata Lin. } & \text { Capo } \\ \text { chilensis Schleg. } & \text { Chili } \\ \text { valdiviensis. } & \text { Valdivia } \\ \text { Smithii D. B. } & \text { Capo }\end{array}$

\section{Venenifera}

(Proteroglypha et Solenoglypha D. B.)

$$
\text { a. MARINA }
$$

18. HYDROPHINA.

Platurus Latr.

fasciatus Daud. Mare indico

Fischeri.

Pelamis Daud.

bicolor Daud. Mare indico

var. sinuata $D$. B. Sumatra

Hydrophis Daud.

schistosa Daud. Golfo diBeng.

pelamidoides Schleg. Malabar,

striata Schleg. Giava 
fasciatus $\boldsymbol{D} . \boldsymbol{B},\left(\operatorname{Schn.9)} \mathrm{N}^{\text {a }}\right.$ Caledonia nigrocincta Daud. Mar. delle In. gracilis Schleg. Costa di Giava controversa.

spiralis D. B. (Shaw.?) Giava microcephalus Schm. Pondichery

\section{b. TERRESTRIA}

\section{ELAPINA.}

\section{Elaps Schneid.}

corallinus Lin. Brasile Mess. var. circinalis $D . B$. Marcgravii Merr. Colombia interruptus. Caracas psyche Merr. Surinam ornatissimus.

fulvius $\boldsymbol{L}$.

lemniscatus Lin. Brasile decussatus $D$. B. Cayenne elegans. diastema $D . B$ Messico decoratus. frontalis $D . B$. Columbia

(Marcgravii Neuw.) surinamensis Cuv. Surinam

lacteus $\boldsymbol{L}$. (hygeiae Merr.)

Aspidelaps. Fitz.

lubricus Laur. Capo

(Elaps lubricus D. B.)

Trimeresurus Lacép.

porphyreus Shaw. Nuova Oland. Alecto Wagl.

curta Schleg. coronata Schleg. Gouldii Gray. bungaroides Schleg. Nuova Oland. Sepedon Merr.

haemachates Merr. Capo Causus Wagl. rhombeatus Licht. Capo
Bungarus Daud. annularis Daud. Giava * semifasciatus Kuhl. , arcuatus D. B. Bengala coeruleus Schneid. $\quad$

Chalinophorus semidoliatus. Giava

Naja Laur. tripudians Laur. Giava haje Lin. Egitto nigricollis (Schleg)

20. VIPERINA.

Dendroaspis Schleg. Iamessonii Trail. Is. S. Thoma Acanthophis Daud.

cerastinus Daud. Nuova Oland. Vipera Laur.

berus Lin.

Lombardia

(Pelias berus Merr.)

var. prester $\boldsymbol{L} . \quad$ Stiria

Redii Laur. (aspis Lin) Padova

ammodytes $L$. Dalmazia

Echidna Merr.
arietans
Capo
nasicornis Merr.
Gabon

(Vip. hexacera D. B.)

gabonica $D . B$. Gabon

lebetina Forsk. (non L.) Cipro

(mauritanica Guichen). elegans Daud. Bengala

Cerastes Wagl.

aegyptiacus $L$. Cairo

lophophrys Cuv. Mozambico

Echis Merr.

carinata Merr. Egitto

\section{GROTALINA.}

Crotalus. Lin. durissus Lalr. America selt. 
adamanteus Beauv. Messico (rhombifer Latr.)

horridus $L$.

Brasile

lugubris.

Messico

miliarius $\boldsymbol{L}$.

America sett.

var. tergeminus Say. America sett. Lachesis Daud.

muta $\boldsymbol{L}$.

Surinam

Trigonocephalus $0 p p$.

piscivorus Lacép. Nuova Orl.

contortrix $\boldsymbol{L}$. Georgia

Blomhoffii Boie. Giappone

hypnale Merr. Ceylon

Leiolepis $D$. $B$.

rhodostoma Reinw. Giava
Bothrops Wagl.

lanceolatus Merr. Antille

atrox Lin. Brasile

var. Neuwiedii Wagl. "

jararaca Neuw. "

Castelnaudi $D . B$.

alternatus $D . B$. America mer.

ocellatus.

Africa occid.

Schlegelii Berth. Brasile

nigromaculatus Wagl. Ceylon

Atropos Wagl.

puniceus Reinw. Giava

undulatus.

Messico

Tropidolaemus Wagl.

Wagleri Boie.

Sumatra, Ma-

nilla

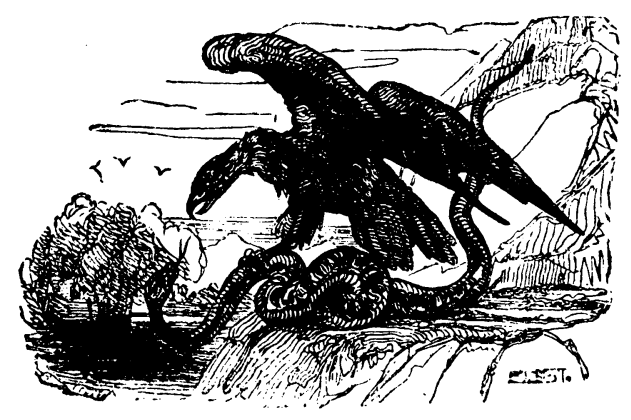




\section{A M P H I B I A}

\section{OPHIOSOMA}

\section{COECILIAE}

Coecilia Wagl.

Lumbricoidea Daud. Surinam albiventris Daud. compressicauda $D$. B. Cayenne Siphonops Wagl.

$\begin{array}{ll}\text { annulatus Mik. } & \text { Bahia } \\ \text { mexicanus D. B. } & \text { Messico }\end{array}$ Epicrium Wagl.

glutinosum $L . \quad$ Calcutta

\section{ANURA}

\section{RANAE}

Pseudis Wagl.

paradoxa $L$.

(Merianae D. B.)

Oxyglossus Tschud.

lima Tschud.

Giava

Physodes (Fitz. Tschud.)

albiventris (Fitz. Tsch.) Brasile

Hyobates (Fitz. Tschud.)

fuscomaculatus (Fitz. Tsch.) Brasile

Rana $\boldsymbol{L}$.

Leschenaultii $D . B$. Mana
esculenta $L$ Lomb., Dalm.
viridis Roesel.
var. ridibunda Pall. Hungaria

- japonica Schleg. Giappone

mascareniensis $D$. B. Mana

halecina Kulm. Georgia Mess.

Savignyi.

Egitto

temporaria $L$.

Lombardia

sylvatica Leconte. America selt. mugiens Catesb. Texas

clamata Daud. America sett.

tigrina Daud. Giava

grunniens Daud.

fuscigula $D . B$. Capo

Delalandii $D . B$.

fasciata Boie.

oxyrhyncha (Schleg).

Cystignathus Wagl.

ocellatus Wagl. Brasile

typhonius Daud. "

labyrinthicus Spix.

Bibroni Tschud. Chili

nodosus $D . B$.

sylvestris (Schleg)

Physolaemus (Fitz. Tschud.)

ephippiger (Fitz. Tsch.) Brasile

Cuvieri (Fitz. Tsch.) ,

albonotatus (Fitz. Tsch.)

Discoglossus Otth. pictus Oth. Sardegna

Ceratophrys Boie.

cornuta $\boldsymbol{L}$.

Brasile

(dorsata Neuw).

Boiei Neuw.

Pyxicephalus Tschud.

Delalandii D. B. Capo

fuscomaculatus (Schleg.) Dekan

Calyptocephalus $D$. $B$.

Gayi $D$. B.

Chili

Cycloramphus Tschud.

marmoratus D. B. Guascona

Megalophrys Kuhl.

montana Kuhl. Giava

Scaphiopus Holbr.

solitarius Holbr. Georgia

spec.

Messico 


\section{HYLAE}

Litoria Tschud.

Freycineti $D . B$. Nuova Oland. marmorata A. Dum. ,

Lithobates (Fitz. Tschud.) palmipes (Fitz. Tschud.) Brasile

Acris $D . B$.

gryllus Leconte. America sett.

Pickeringii Holbr. Pennsylvania

Limnodytes $D$. B.

erythraeus Schleg. Giava -

chalconotus Schleg. ,

Polypedates Tschud.

leucomystax Grav. Martinica

rugosus D. B. Giava

Ixalus $D$. $B$. aurifasciatus Schleg. ,

Eupodion (Fitz. Tschud.)

triste (Fitz. Tschud.) Brasile

Eucnemis Tschud.

madagascariensis $\boldsymbol{D}$. $\boldsymbol{B}$. Madagascar

var. punctulata $B i b r$.

Fornasinii Bianconi. Mozambico

Hylambates A. Dum.

maculatus A. Dum. Zanzibar

Rhacophorus Kuhl.

Reinwardtii Boie. Giava

Trachycephalus Tsch.

geographicus $D$. B. Messico

marmoratus D. B. Cuba

Hyla Laur.

palmata Daud. Brasile

Doumercii D. B. . Surinam

cinerascens Spix. Brasile

Leprieuri $D$. B. Cayenne

adspersa.

Brasile

albomarginata Spix. $\quad$

Langsdorfii $D$. B. "

venulosa Daud.

Baudini D. B. Tabasco

pumila $D . B$ Brasile

bucephala (Schleg). "

versicolor Daud. Georgia scripta (Fitz.) Brasile

cyanea Daud. Nuova Oland.

arborea $L$ Lombardia

(viridis Laur.)

var. japonica. Giappone

lateralis Daud. Georgia, Mes.

pulchella $D$. B. Cordilliere

rubra Daud. Brasile

var. X signata Spix.

decussata (Fitz. Tsch.) •

Ewingii $D . B$. Nuova Oland.

marsupiata D. B. Quito

citropa Per. et Les. Nuova Oland.

aurea Less.

jacksoniensis D. B. , ,

boans Daud. Surinam

femoralis Holbr. America sett.

leucophyllata Beiris. Brasile

spec. Messico

spec. Nuova Olanda

spec. China

Microhyla Tschud.

achatina Tschud. Giava

bengalensis. Bengala

Hylodes Fitz.

martinicensis Tschud. Martinica

laticeps A. Dum. Messico

Phyllomedusa Wagl.

zo bicolor Bodd. Brasile

Elosia Tschud.

nasus Licht.

Phyllobates $D$. B.

bicolor D. B. Cuba

melanorhinus Berth. Popayan

\section{BUFONES}

Hylaemorphus (Fitz. Tsch.)

plutonius (Fitz. Tsch.) Brasile

Dendrobates Wagl.

tinctorius Schneid. Brasile

quinquevittalus (Fitz. Tsch). .

histrionicus Berth. Veragoa

obscurus D. B. 
pictus $D . B$. Chili

Rhinoderma $D$. $B$.

Darwini $D . B$.

Buff Laur.

Leschenaultii $\boldsymbol{D}$. B. Venezuela asper Gravenh. Giava

vulgaris Laur. Lombardia

var. japonica Schleg. Giappone

- palmarum Cuv. Sicilia

chilensis D. B. Chili

viridis Laur. Lombar. Dal. var. calamita Laur.

pantherinus Boie. Egitto

musicus Bosc. America sett.

americanus Leconte. Georgia

var. quadripunctata.

scaber Daud.

Giava

agua Laur.

Brasile

cinctus Neuw.

(melanotis D. B.)

peltocephalus Tsch. Cuba

biporcatus Schleg. Giava

strumosus Daud. Côte ferme

margaritifer Laur. Brasile

guineensis Schleg. Guinea

celebensis Schleg. Celebes

anomalus. Brasile

spec. China

Physalus

fuscorufus. Quito

Phryniscus Wiegm.

nigricans Wiegm. Brasile

australis Gray. Nuova Oland.

ignescens Cor. Quito

spec.

spec.

Brachycephalus Fitz.

ephippium Spix. Cayenne

Hylaedactylus Tsch.

baleatus Tsch.

spec.

Engystoma Tsch.

ovale Schneid.

Colombia

Nuova Oland.

carolinense Holbr.

Buenos Ayres

Giorgia rugosum D. B. Messico

microps $D$. B. Brasile

guineense (Schleg). Guinea

inconspicuum. Messico

Eupomplyx (Fitz. Tschud.) marmoratus (Fitz. Tsch.) Brasile

Paludicola Wagl.

albifrons Spix.

Breviceps Merr.

gibbosus $\boldsymbol{L}$.

Capo

mossambicus Pet. Mozambico

Rhinophrynus $D$. B.

dorsalis $D$. B. Messico

BOMBINATORES.

Pelodytes Fitz.

punctatus Daud. Francia

Alytes Wagl.

obstetricans Daud. Baviera

Pelobates Wagl.

fuscus Laur. Vienna

Bombinator.

igneus

Lombardia

PIPAE.

Dactylethra Cuv.

capensis Cuv. Capo

spec.

Abissinia

Pipa Laur.

americana Laur. Surinam

III. URODELA $D, B$.

(Delesura)

\section{SALAMANDRIDA.}

Salamandra Wurffb.

maculosa Laur. Lombardia

atra Laur.

Tirolo, Stiria
Salamandrina Fitz.

perspicillata Savi. Genova, Nervi 
Pleurodeles Michahell.

Waltlii Michah. Spagna

Cylindrosoma Tsch.

longicaudatum Green. Georgia

Plethodon Tsch.

glutinosum Tsch. Georgia variolosum Tsch. America sett. fuscum Green.

erythronotum Green. Georgia

Geotriton C. Bonap. fuscus Gené. Nervi

Pseudotriton Tschud.

(Bolitoglossa D. B.)

ruber Pal. d. Beauv. Georgia bilineatus Green. Carolina

mexicanus Cuv. Messico

(0edipus platydactylus $T s c h$.

Hynobius Tschud.

(Ellipsoglossa D. B.)

naevius Schleg.

nebulosus Schleg.

Giappone

Ambistoma Tschud. punctatum $\boldsymbol{L}$. nigrum Green. Georgia fasciatum Harl. Carolina tigrinum $\mathrm{Harl}$. Onychodactylus Tsch.

Schlegelii Tschud. Giappone

Cynops Tsch.

(S. unguiculata Schleg.)

subcristatus Schleg. Giappone

Triton Laur.

cristatus Laur. Lombardia marmoratus Latr. Francia mer.

taeniatus Schneid. Lombardia (punctatus Latr.) alpestris Schneid. Appennini neglectus.

palmatus Schneid. Lombardia

symmetricus Harl. America sett. dorsalis Harl.

Euproctus Gené.

Rusconi Gené. Sardegna

Xiphonurus Tsch.

Ièffersoni Green. California

\section{AMPHIUMIDA.}

Amphiuma Harlan.

tridactylum Harl. America sett.

Menopoma Harl.

alleghanensis Harl. America sett.

\section{PROTEIDA.}

Siredon Wagl.

pisciformis Shaw. Messico

(Humboldii D. B.)

Menobranchus Harl.

lateralis Gay. America sett.

Proteus Laur.

anguinus Laur. Carniola

var. Laurenti Fitz. ,

๑ Schreibersii Fitz. ,

- Haidingeri Fitz. ,

- Zoysii Fitz.

- Freyeri Fitz.

๖ Carrarae Fitz. Dalmazia

Siren $L$.

lacertina $\boldsymbol{L}$.

Carolina

var. striata Leconte , 


\section{INDEX GENERUM}

arron $\Omega \notin 8<0$ vornos

T. Testudinata - S. Sauria - 0. Ophidia - A. Amphibia.

o. Ophiosoma - a. Anura - u. Urodela.

0. Ablabes . . . . . .pag. 46

S. Ablepharus . . . . . , 42

S. Acanthodactylus . . ., 40

0. Acanthophis . . . . . , 50

S. Acontias . . . . . . , 42

S. Acrantus . . . . . . 40

A. a Acris . . . . . . . \53

S. Agama . . . . . . $\quad .39$

0. Alecto . . . . . . . „50

S. Alligator . . . . . . , 36

A. a Alytes . . . . . ., 54

A. u Ambystoma . . . . . , 5丂

0 . Amblycephalus . . . . " 48

S. Ameiva . . . . . . „40

S. Amphisbaena . . . . , 41

0. Amphiesma . . . . . 47

A. u Amphiuma . . . . . \55

S. Anguis . . . . . . , 42

0. Anholodon . . . . . \$47

S. Anisoterma . . . . . , 42

S. Anolis . . . . . . . , 38

0. Anomolepis . . . . . \43

0. Aplopeltura . . . . . 48

S. Aporomera . . . . . , 40

0. Aspidelaps . . . . . , 50

0. Aspidura . . . . . . , 44

0. Atropos . . . . . . "51

S. Basiliscus. . . . . . , 38

0. Boa . . . . . . . \44

0. Boaedon . . . . . . \$47

A. u Bolitoglossa . . . . . \ 5ั

A. a Bombinator . . . . . , 54

0. Bothrops . . . . . „51
A. a Brachycephalus . . . .pag. 54

S. Brachylophus . . . . , 38

0. Brachyorrhos . . . . . 47

0. Brachyrrhyton . . . . , 49

A. a Breviceps. . . . . . $\$ 54$

0. Bucephalus . . . . . , 48

A. a Bufo . . . . . . . \$54

0. Bungarus. . . . . . ๑ 50

0. Calamaria. . . . . . , 4/4

0. Calopisma . . . . . , 46

S. Calopistes . . . . . 40

S. Calotes '. . . . . . , 39

A. a Calyptocephalus . . . . , 52

0. Campsosoma . . . . . \46

0. Carphophis . . . . . , 44

0. Causus . . . . . . „ 0

0. Celuta. . . . . . . „44

S. Centropyx . . . . . , 40

S! Centrura . . . . . , 38

0. Cephalolepis . . . . . \ $\mathbf{4 3}$

0. Cerastes . . . . . . \50

S. Ceratophora . . . . . , 39

A. a Ceratophrys . . . . . , 52

0. Cerberus . . . . . . \$49

S. Chalcides. . . . . , $4 \mathbf{4 1}$

0. Chalinophorus . . . . \50

S. Chamaeleo . . . . . , 37

S. Chamaesaura . . . . \ 41

T. Chelodina . . . . . , 36

T. Chelonia . . . . . . , 36

T. Chelydra . . . . . . , 36

T. Chelys . . . . . . , 36

S. Cherdaleus . . . . . , 39 
0. Chersydrus . . . . . pag. 4.3 0. Dryophis . . . . . .pag. 48

0. Chilabothrus. . . . . . 44 0. Dryophylax . . . . . „ 49

0. Chlorosoma . . . . . \45 0 . Echidna . . . . . . $\$ 80$

0. Chrysopelea . . . . . \$49 0 . Echis . . . . . . . \$50

T. Cinosternon . . . . . „36 S. Ecphymotes . . . . . „39

T. Cinixys . . . . . . \$35 0 . Elaphis . . . . . . , 46

T. Cistudo . . . . . . \$35 0 . Elapoides. . . . . . 44

S. Cnemidophorus . . . . \40 0 . Elapomorphus . . . . \48

0. Cochliophagus . . . . \$ 47 0. Elaps . . . . . . . \$50

A. o Coecilia . . . . . . \ 52 A. u Ellipsoglossa . . . . . \ 355

0. Coelopeltis . . . . . \49 A. a Elosia . . . . . . . \53

S. Coleonyx. . . . . . 37 T. Emyda . . . . . . 36

0. Coluber . . . . . . \44 T. Emys . . . , . . . „35

0. Coloburus . . . . . \43 T. Emysaurus . . . . . ”36

0. Conocephalus. . . . . \$44 0 . Enicognathus . . . . \$ 46

S. Copeoglossum . . . . ๑41 A. a Engystoma . . . . . \$ 54

0. Coronella. . . . . . \44 S. Enyalius . . . . . . „38

0. Coryphodon . . . . . „44 0. Enygrus . . . . . . \$ 44

S. Crocodilurus. . . . . \$40 0 , Epicarsius . . . . . \$44

S. Crocodilus . . . . . , 36 0. Epicrates. . . . . . , 43

0. Crotalus . . . . . . . 50 A. o Epicrium . . . . . . , 52

T. Cryptopus . . . ., 36 S. Eremias . . . . . . 40

S. Cyclodus . . . . . . \$42 0 . Erythrolamprus . . . . \$48

0. Cyclochorus . . . . . \$47 4 . Eryx . . . . . . . . 43

A. a Cycloramphus . . . . „52 S. Espondylus . . . . . 41

S. Cyclura . . . . . . \$38 0 . Etairejus . . . . . . , 46

0. Cylindrophis . . . . . \$43 A, a Eucnemis . . . . . . " 53

A. u Cylindrosoma . . . . \$ 54 0. Eucritus . . . . . . , 44

A. u Cynops . . . . . . \$55 0. Eugnathus . . . . . \$47

A. a Cystignathus . . . . \$52 S. Eumeces . . . . . . \$41

A. a Dactylethra . . . . . \$ 54 0. Eunectes . . . . . . , 44

0. Dendroaspis . . . . . „50 A. a Eupodion. . . . . . \$53

A. a Dendrobates. . . . . , 53 A. a Eupomplyx . . . . . , 54

0. Dendrophis . . . . . „45 S. Euprepes. . . . . . „41

S. Dibamus . . . . . . \42 A. u Euproctus . . . . . „55

S. Diploglossus . . . . . „ $4 \mathbf{1}$ 0. Eurostus . . . . . . , 49

0. Dipsadomorus . . . . \$48 S. Gavialis . . . . . . , 37

0. Dipsas . . . . . . \49 A. u Geotriton . . . . . ๑5̈

A. a Discoglossus . . . . . " 52 S. Gerrhonotus . . . . . , 41

0. Dochis . . . . . . "44 S. Gerrhosaurus . . . . 41

S. Doryphorus . . . . . " 39 S. Gongylus . . . . . . 41

S. Draco. . . . . . . \39 S. Grammatophora . . . , 39

0. Dromicus. . . . . . , 46 S. Gymnodactylus . . . . , 37

0. Dryinus . . . . . . \$ 48 S. Gymnophthalmus . . . , 12 
T. Gymnopus. . . . . pag. 36

0. Hapsidrophis .

0. Helicops

S. Hemidactylus

S. Hemiergus

0. Hemiodontus .

0. Herpetodryas .

0. Heterodon

S. Heteromeles .

S. Heteropodium

S. Heteropus

0. Himantodes

S. Holbrookia

S. Holotropis

0. Homalocranion

0. Homalopsis

0. Homalosoma .

T. Homoeopus .

S. Hoplocercus .

0. Hydrophis

0. Hydrops .

A. a Hyla

A. a Hylaedactylus

A. a Hylambates

A. a Hylodes

A. u Hynobius .

A. a Hyobates .

S. Hyperanodon

S. Hypsibatus

0. Hypsirrhina

S. Hysteropus

S. Iguana

0. Ischnognathus

S. Istiurus

A. a Ixalus .

S. Lacerta

0. Lachesis

S. Laemanctus . . . . ., 38

0. Lamprophis . . . . . "47

0. Langaha . . . . . . " 48

S. Leiolepisma . . . . . \42

0. Leiolepis . . . . . . \$51

S. Leiosaurus
- 45

- 46

37

42

48

, 45

46

42

- 42

, 42

49

39

- 38

48

49

44

\35

, 39

" 49

47

- 53

54

" 53

\$3

- 53

52

38

38

49

42

38

47

, 39

53

40

51

38

8

2

38
S. Lepidophymus

S. Lepidosternon

pag. 41

0. Leptoboa .

0. Leptognathus

0. Leptophis .

S. Lialis .

0. Liasis . . . . . . . " 43

A. a Limnodytes . . . . . „53

0. Liophis . . . . . . " 47

A. a Lithobates . . . . . , 53

A. a Litoria . , . . . . , 53

S. Lophyrus . . . . . . , 39

0. Lycodon . . . . . . 447

0. Lycognathus . . . . . 48

S. Lygosoma . . . . . " 42

S. Lyriocephalus . . . . " 39

A. a Megalophrys . . . . . \52

A. u Menobranchus . . . . " 5 5

A. u Menopoma . . . . . " 55

A. a Microhyla. . . . . . \ 53

S. Microlophus . . . . . \$39

S. Moloch . . . . . . $\$ 40$

0. Morelia . . . . . . „43

0. Naja . . . . . . . ๖ 50

S. Norops . . . . . . \38

0. Odontomus . . . . ., 48

0. Oligodon . . . . . . , 44

0. Oligotropis . . . . . . 49

0. Onychocephalus . . . . 43

A. u Onychodactylus . . . . , 55

0. Opetiodon . . . . . \ 48

S Ophiomorus . . . . . , 42

S. Ophiops . . . . . . , 40

S. Ophisaurus . . . . . \$ 41

0. Ophites . . . .. . . , 47

S. Ophryoessa . . . . . „ 38

0. Ophthalmidion . . . . , 43

S. Oplurus . . . . . . , 39

0. Osceola . . . . . . " $4 / 4$

0. Oxybelis . . . . . . $\$ 49$

A. a Oxyglossus . . . . . \ 52

0. Oxyrrhopus . . . . ., 49

A. a Paludicola . . . . . ; 54

0. Pareas . . . . . . $\$ 48$ 
0. Pelamis

pag. 49

0. Pelias .

0. Python

pag. 43

A. a Pelobates .

A. a Pyxicephalus

52

A. a Pelodytes.

0. Rachiodon . . . . . \$47

T. Pelomedusa . . . . . 》36

T. Pentonyx . . . . . „36

0. Periops . . . . . . , 46

0. Petalognathus . . . . , 47

A. a Phryniscus . . . . . " 54

S. Phrynocephalus . . . . $\quad ₫ 39$

A. a Racophorus . . . . . \53

A. a Rana . . . . . . . " 52

0. Rhabdion . . . . . . " 44

0. Rhabdosoma . . . . . 44

0. Rhinechis . . . . . . , 46

0. Rhinobothrium . . . . 49

S. Phrynosoma . . . . . , 39

A. a Phyllobates . . . . . $\$ 53$

S. Phyllodactylus . . . . , 37

0. Rhinocleis . . . . . , 46

A. a Rhinoderma . . . . . \53

A. a Rhinophrynus . . . . , 54

A. a Phyllomedusa . . . . „53

A. a Physalus . . . . . . "54

0. Rhinosimus . . . . 48

0. Rhinostoma . . . . . ๑ 48

A. a Physodes .

A. u Salamandra . . . . . , 544

A. a Physolaemus . . . ., 52

A. u Salamandrina . . . . .54

0. Pilidion . . . . . . „ 43

A. a Pipa . . . . . . . \$ 54

0. Pituophis . . . . . . \46

T. Platemys . . . . . . " 36

0. Platurus . . . . . ., 49

S. Platydactylus. . . . . „37

0. Plectrurus . . . . . ” 43

0. Plesius . . . . . . " 44

S. Plestiodon . . . . . " 42

A. u Plethodon . . . . . " $\mathbf{3 4}$

S. Salvator . . . . . . \$40

A. a Scaphiopus . . . . . \52

S. Scapteira . . . . . . 40

S. Scelotes . . . . . . \42

S. Scincus . . . . . . 41

0. Scolecophis . . . . . " $4 / 4$

0. Scydropus . . . . . 47

0. Scytale . . . . . . " 48

0. Sepedon . . . . . . " 50

S. Seps . . . . . . . $\$ 42$

0. Simotes . . . . . . \$ 45

A. u Pleurodeles . . . . . „ 54 A. o Siphonops . . . . . " 52

S. Podinema. . . . . . \$40

A. u Siredon . . . . . . $\$ 5 \%$

T. Podocnemis . . . . . " 36

S. Polychrus . . . . . \38

A. a Polypedates . . . . . \$ 53

A. u Siren . . . . . . . „55

S. Sitana. . . . . . . $\quad$ ๖ 39

T. Sphargis . . . . . . , 36

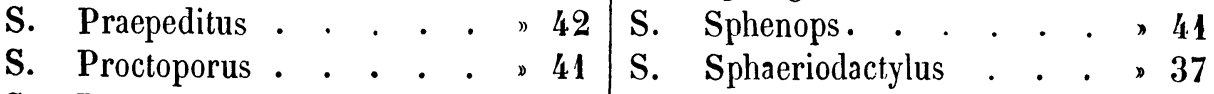

S. Proctotretus . . . . . \$38 0 . Spilotes . . . . . . 45

A u Proteus . . . . . . , 55 T. Staurotypus . . . . . \36

0. Psammophis . . . . . " 48 S. Stellio. . . . . . . ” 39

S. Psammodromus . . . . " 40 S. Stenodactylus . . . . " 37

A. a Pseudis . . . . . . " 52 0. Stenostoma . . . . . „43

0. Pseudoperiops . . . . " 46 0. Stenorrhina . . . . . ” 48

S. Pseudopus . . . . . \$41 0 . Storeria . . . . . . "47

A. u Pseudotriton . . . . . „5: T. Sternotherus . . . . . „36

S. Ptyodactylus . . . . , 37 0. Stremmatognathus . . . , 47

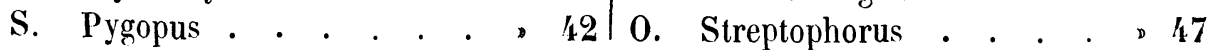


S. Strobilurus . . . . pag. $\left.39\right|^{0}$. Tropidonotus . . . pag. 45

S. Tachydromus . . . . , 40 S. Tropidolepis . . . . . , 39

0. Tarbophis . . . . . , 48 S. Tropidolepisma . . . . , 42

0. Telescopus . . . . ., 490 . Tropidopeltis . . . ., 47

T. Testudo . . . . . . , 35 0 . Tropidophis . . . . . , 44.

S. Tetradactylus . . . . , 42 S. Tropidosaura . . . . , 40

0. Tomodon. . . . . . , 48 S. Typhline . . . . . . , 42

0. Tortrix . . . . . . , 43 0. Typhlops . . . . . . , 43

A. a Trachycephalus . . . . , 53 S. Uromastix . . . . . , 39

S. Trachysaurus . . . . , 42 S. Urostrophus . . . . . , 38

0. Tragops . . . . . . , 48 S. Varanus . . . . . . , 37

0. Tretanorhinos. . . . , 460 . Vipera. . . . . . . , 50

0. Triglyphodon . . . . , 49 0. Xenoderma . . . . . , 43

0. Trigonocephalus . . . . , $\mathbf{5 1} 0$. Xenodon . . . . . . , 46

0. Trimeresurus . . . . , 50 0. Xenopeltis . . . . . , 43

T. Trionyx . . . . . . , 36 A. u Xiphonurus . . . . . , 55

A. u Triton . . . . . . , 550 0. Xiphosoma . . . . . , 43

S. Trogonophis . . . . . , 410 . Zamenis . . . . . . , 46

0. Tropidolaemus . . . . , 51 S. Zonurus. . . . . . 40

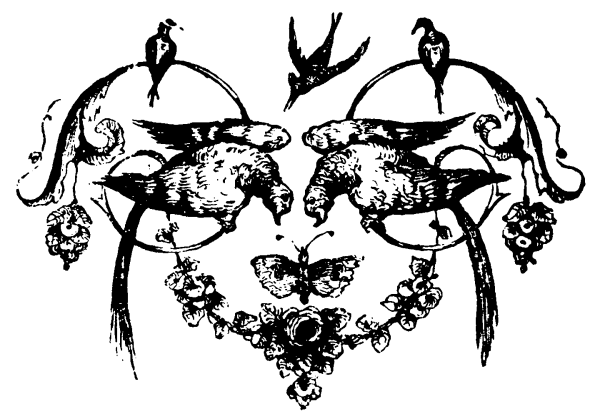





\section{COLLCAZIONE DEGLI OGGETTI ESPOSTI}

\section{Vertebrati.}

I Golleriar: Orhammiferi ed Olccelli: II." Galleria: Rattili e Pesci.

$$
\text { bttigue alla II Calleiea }
$$

1.' Etanza: Raccolta osteologica.

II. Cltance: Araniv fofoili di ORammiferi ed Ulacelli, Rettilive Pesci prep a secce. III" Ćtana: Rettili coesci fofoili.

\section{Invertebrati}

III' Galleria : ousetti ed alkw Clafoi d'a wuestebrati. 1. Clanza: Crostacei.

II. Clanza Conchighic fofoili e Polipari.

IV." Galleria: Conchightie marime, terrestri e Phuviali- Thineralive roccie

Mllima Slana: Eubario e vegetabili foosili.

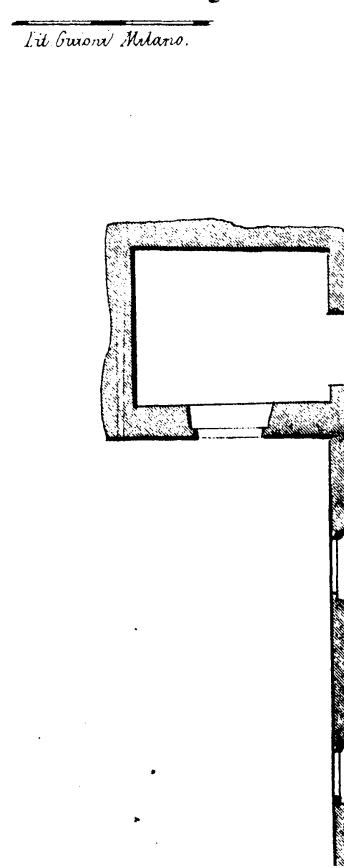




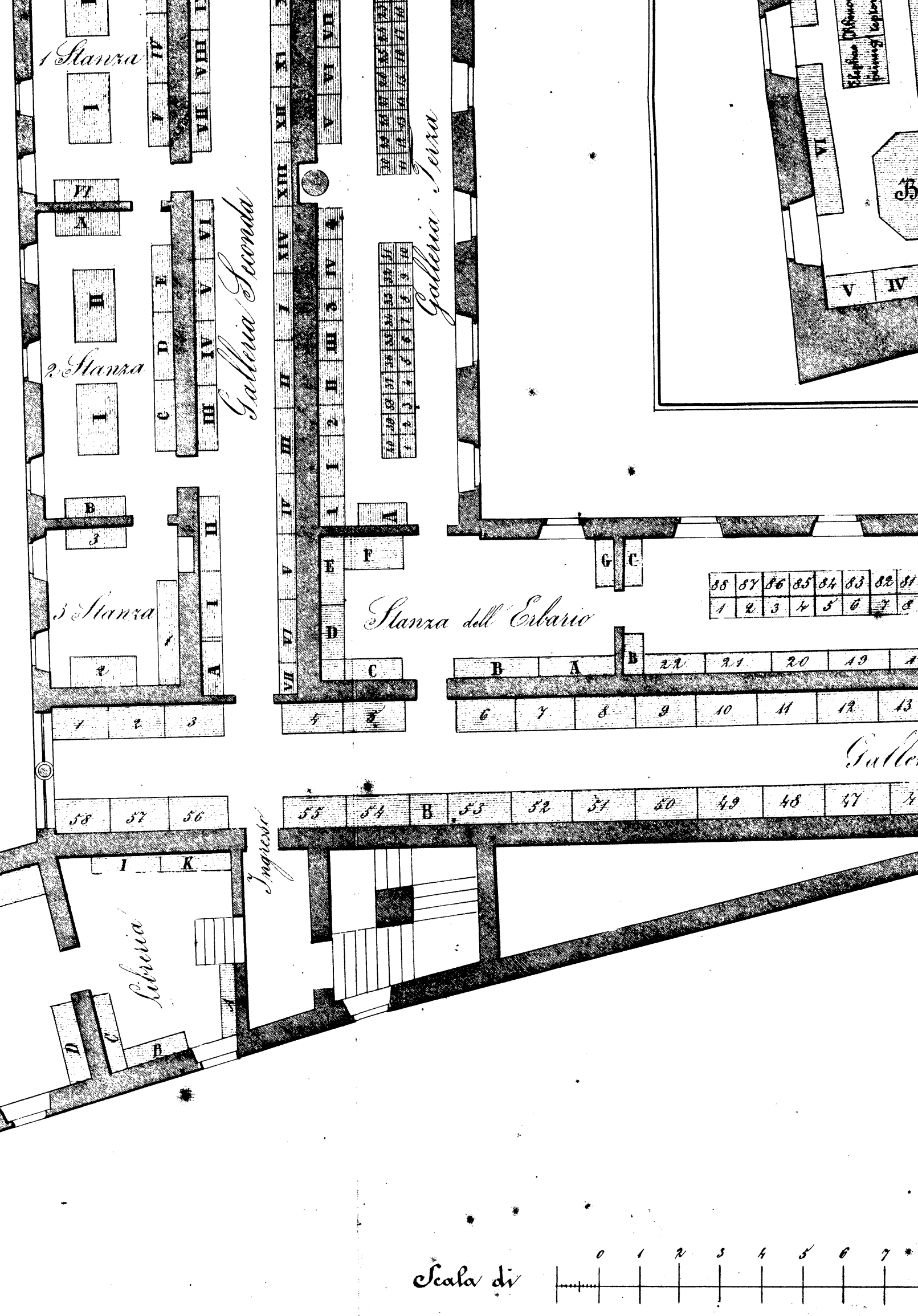




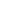




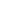




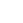



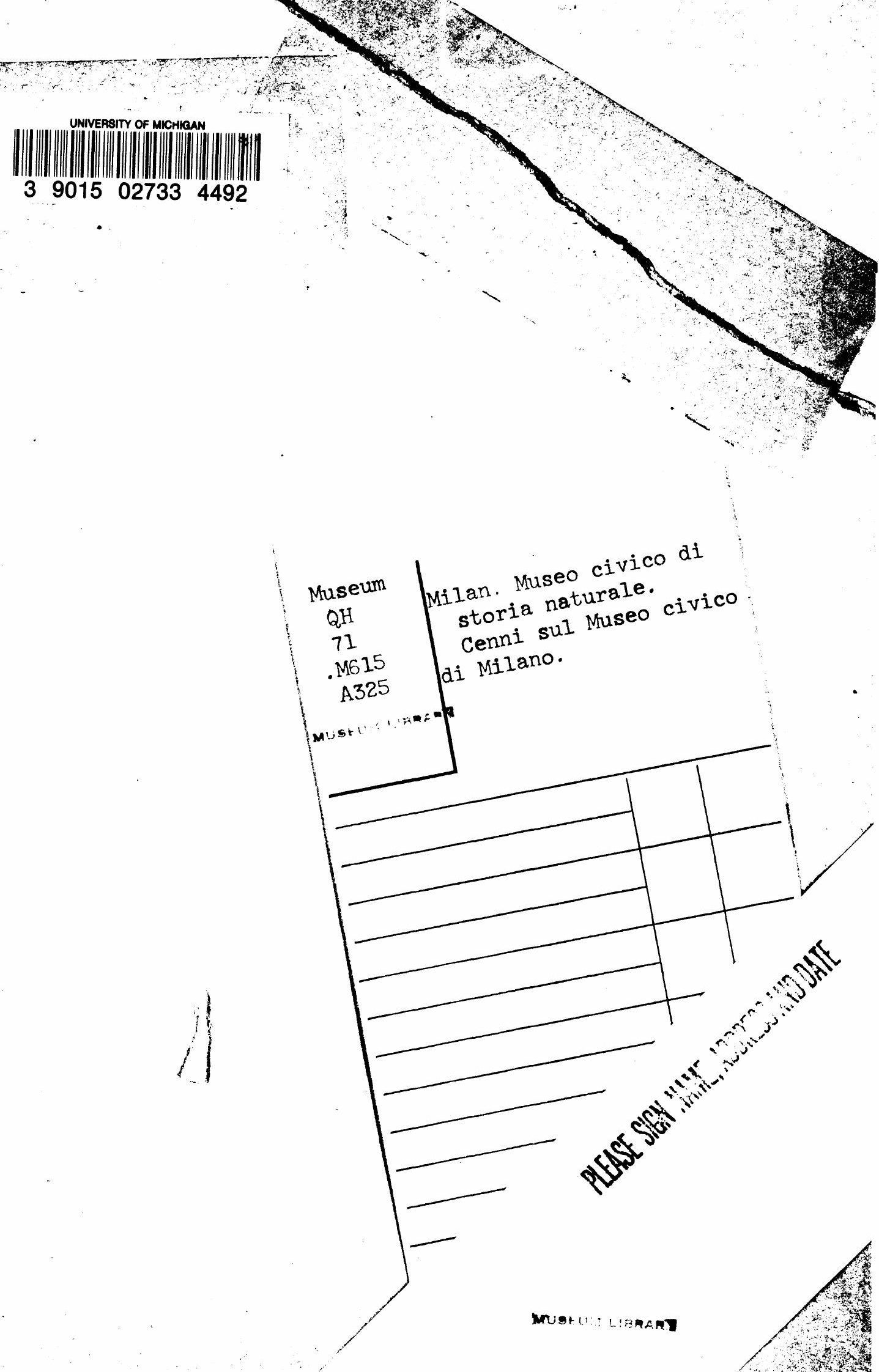


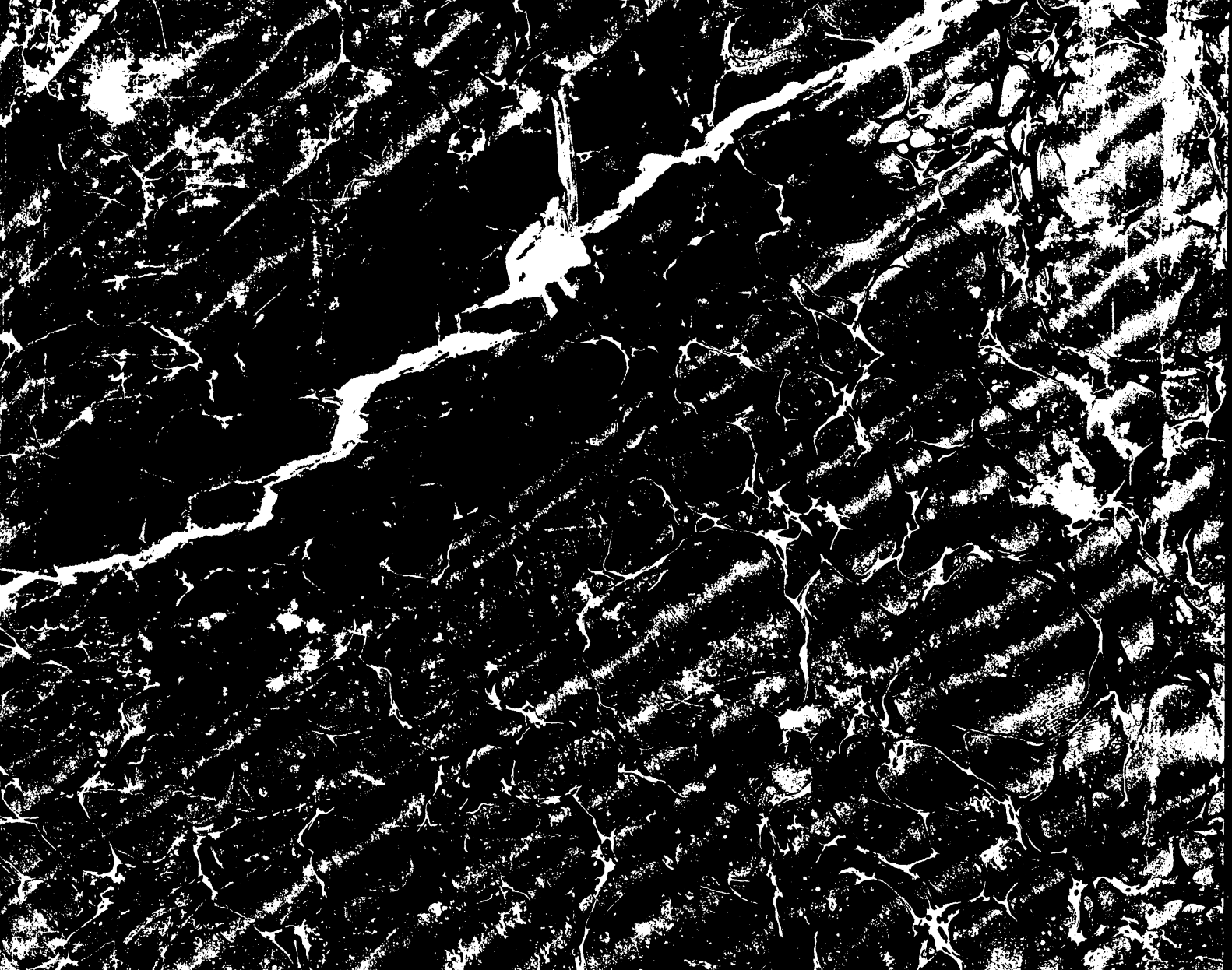

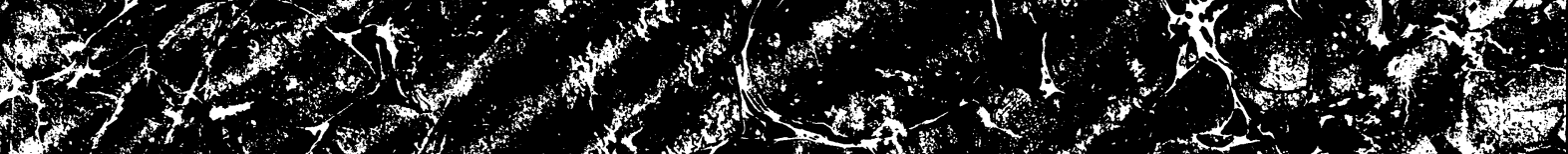

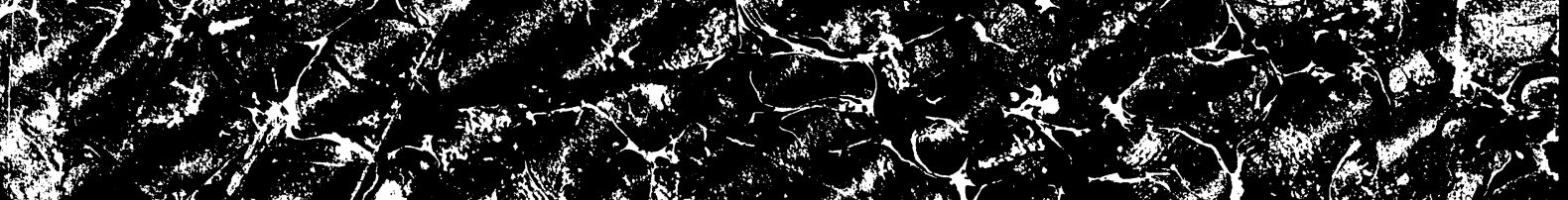

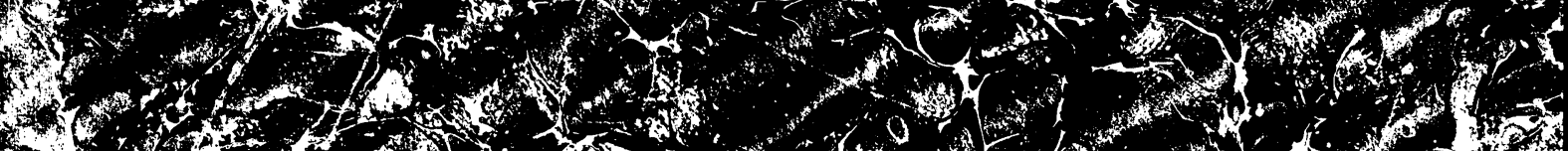
(x)

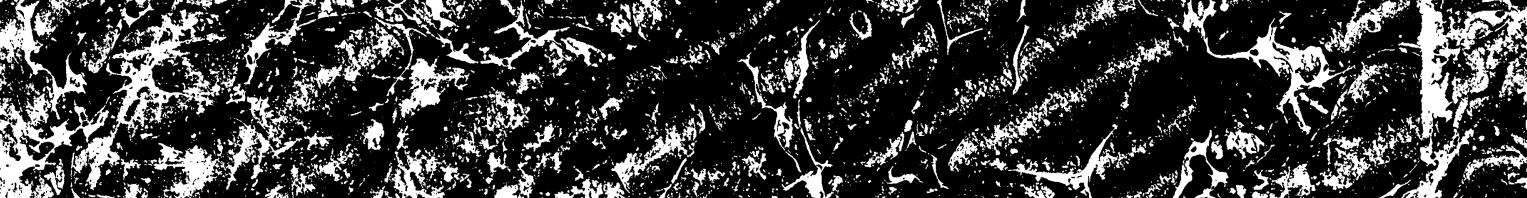

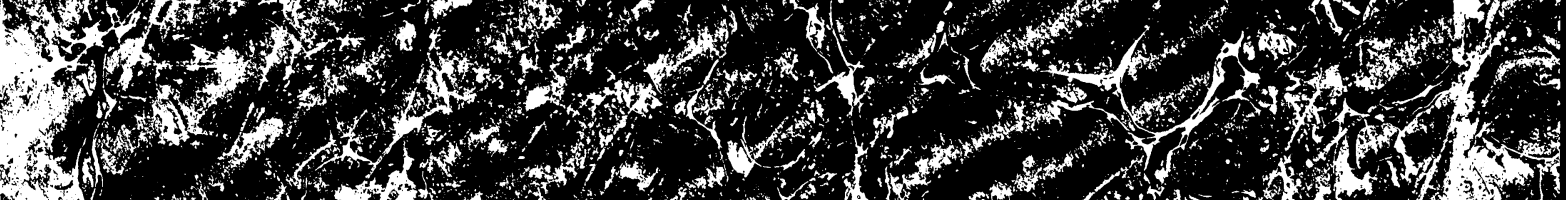

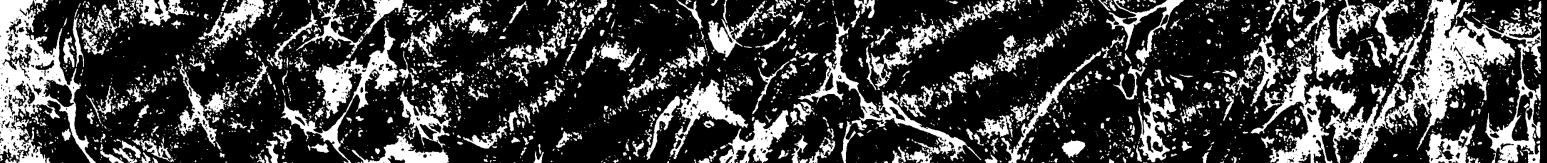

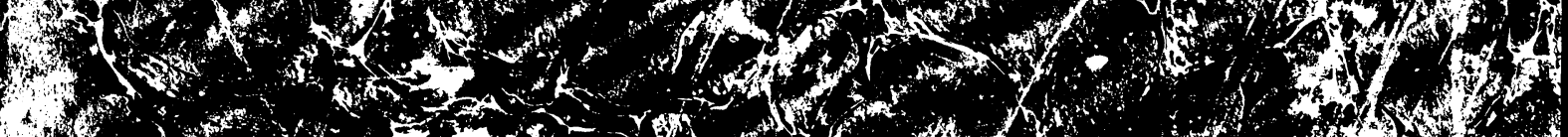

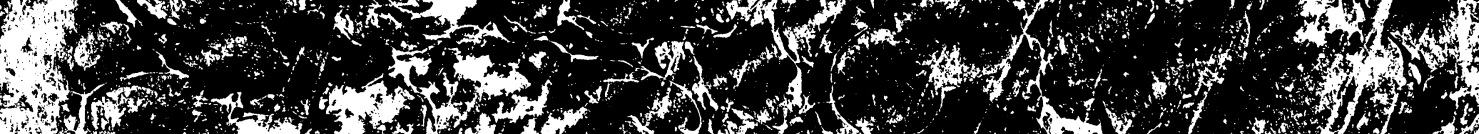

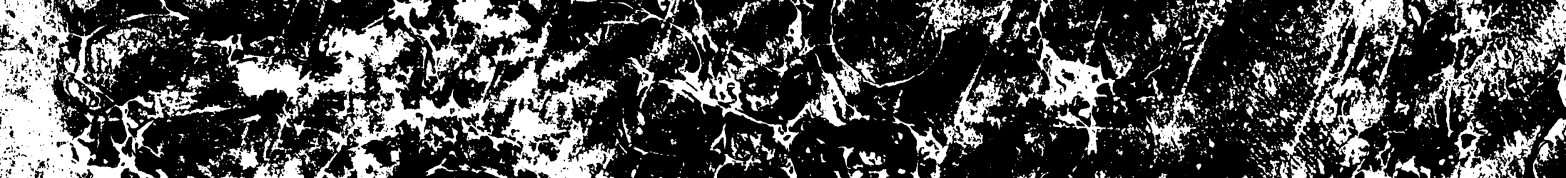

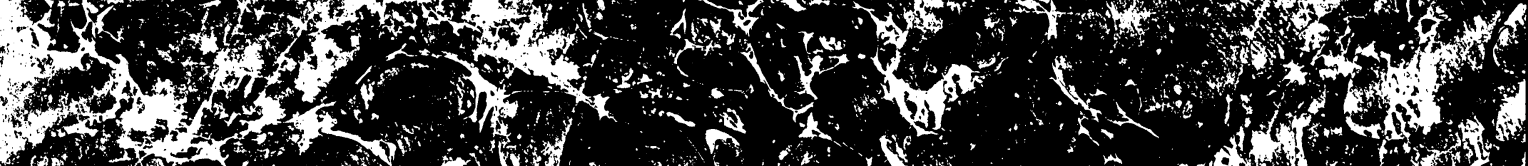

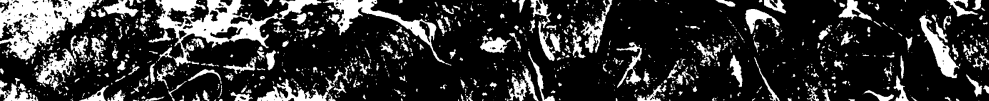

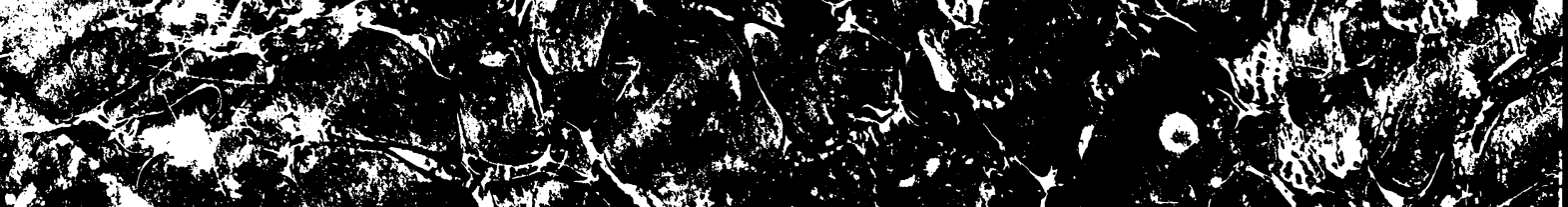

